\title{
Ultrafast Sample placement on Existing tRees (UShER) enables real-time phylogenetics for the SARS-CoV-2 pandemic
}

\author{
Yatish Turakhia $\mathbb{1}^{1,2}$, Bryan Thornlow ${ }^{1,2}$, Angie S. Hinrichs ${ }^{2}$, Nicola De Maio ${ }^{3}$, \\ Landen Gozashti $\mathbb{1}^{1,2,4}$, Robert Lanfear ${ }^{5}$, David Haussler ${ }^{1,2,6}$ and Russell Corbett-Detig ${ }^{1,2,7 凶}$
}

\begin{abstract}
As the SARS-CoV-2 virus spreads through human populations, the unprecedented accumulation of viral genome sequences is ushering in a new era of 'genomic contact tracing'-that is, using viral genomes to trace local transmission dynamics. However, because the viral phylogeny is already so large-and will undoubtedly grow many fold-placing new sequences onto the tree has emerged as a barrier to real-time genomic contact tracing. Here, we resolve this challenge by building an efficient tree-based data structure encoding the inferred evolutionary history of the virus. We demonstrate that our approach greatly improves the speed of phylogenetic placement of new samples and data visualization, making it possible to complete the placements under the constraints of real-time contact tracing. Thus, our method addresses an important need for maintaining a fully updated reference phylogeny. We make these tools available to the research community through the University of California Santa Cruz SARS-CoV-2 Genome Browser to enable rapid cross-referencing of information in new virus sequences with an ever-expanding array of molecular and structural biology data. The methods described here will empower research and genomic contact tracing for SARS-CoV-2 specifically for laboratories worldwide.
\end{abstract}

\begin{abstract}
$\mathrm{n}$ the past year, the SARS-CoV-2 virus quickly spread across human populations worldwide ${ }^{1-3}$. Recent technological advances have enabled rapid and cost-efficient sequencing of the viral genome-over 2,000 groups worldwide have generated 97,733 high coverage whole-genome SARS-CoV-2 sequences that are available on GISAID ${ }^{4}$ as of 24 September 2020. These vast datasets and rapid sequencing turnaround times are enabling a type of 'genomic contact tracing' where genetic similarities between viral genomes isolated for different hosts carry important information about the transmission dynamics of the virus. For example, these data can be used to infer the number of unique introductions of the viral genome in a given area ${ }^{5-11}$ and identify 'transmission chains' among seemingly unrelated infections $s^{12-16}$.

Despite great potential, this unprecedented and ongoing accumulation of sequencing data is overwhelming existing systems for the analysis and interpretation of viral transmission and evolutionary dynamics. In part, this is because typical phylogenetic applications accumulate all of the relevant sequence data before beginning phylogenetic inference. For genomic contact tracing to work effectively, each new viral genome sequence must be contextualized within the entire evolutionary history of the virus rapidly and accurately as it is collected. This could be accomplished by re-inferring the full phylogeny, but with current SARS-CoV-2 datasets containing hundreds of thousands of samples, this takes more than a day even using powerful computational resources. Alternatively, new genome sequences could be contextualized by placing samples onto an existing 'reference phylogeny' and several methods have been
\end{abstract}

developed for this purpose ${ }^{17-20}$. These methods have been used to place new samples onto a phylogeny created from a small subset of available SARS-CoV-2 isolates ${ }^{21}$ and provide regular updates to a global phylogeny of SARS-CoV-2 (ref. ${ }^{22}$ ). Nonetheless, existing algorithms for placing sequences onto reference phylogenies are far too slow to enable real-time genomic contact tracing (Table 1).

Quantification of uncertainty is a fundamental aspect of interpreting phylogenetic inferences ${ }^{23}$ and sample placements onto a reference phylogeny. Nonparametric bootstrapping ${ }^{24}$ has been a cornerstone of phylogenetic inference for decades but this is impractical for the extremely large sample sizes and limited phylogenetic information in SARS-CoV-2 genome isolates. More recently developed methods, such as ultrafast bootstrapping ${ }^{25,26}$, are fast but not applicable to the problem of placing individual samples onto a reference phylogeny. An alternative to these approaches is the approximate likelihood-ratio test ${ }^{27}$ but its computation is prohibitively slow and interpretation challenging. Therefore, quantifying uncertainty in sample placement on reference phylogenies is an important unsolved problem and particularly relevant during this pandemic.

In this work, we describe an efficient method that facilitates rapid, maximum parsimony addition of samples onto an existing phylogeny. We show that our method for placing new genome sequences onto a SARS-CoV-2 phylogeny is orders of magnitude faster than existing approaches and produces highly accurate results. Additionally, we introduce the branch parsimony score (BPS), which is the minimum number of additional mutations

\footnotetext{
'Department of Biomolecular Engineering, University of California Santa Cruz, Santa Cruz, CA, USA. ${ }^{2}$ Genomics Institute, University of California Santa Cruz, Santa Cruz, CA, USA. 'European Molecular Biology Laboratory, European Bioinformatics Institute, Wellcome Genome Campus, Cambridge, UK. ${ }^{4}$ Department of Organismic and Evolutionary Biology and Museum of Comparative Zoology, Harvard University, Cambridge, MA, USA. ${ }^{5}$ Department of Ecology and Evolution, Research School of Biology, Australian National University, Canberra, Australian Capital Territory, Australia. ${ }^{6} \mathrm{Howard} H \mathrm{Hughes}$ Medical Institute, University of California Santa Cruz, Santa Cruz, CA, USA. ${ }^{7}$ National Research University Higher School of Economics, Moscow, Russian Federation.凶e-mail: yturakhi@ucsc.edu; rucorbet@ucsc.edu
} 
Table 1 | Average time and time range required to place one sample and peak memory usage across 20 replicate runs of each placement algorithm

\begin{tabular}{|c|c|c|c|c|}
\hline Program & $\begin{array}{l}\text { Average time to place one } \\
\text { sample }\end{array}$ & $\begin{array}{l}\text { Time range over } 20 \\
\text { replicates }\end{array}$ & $\begin{array}{l}\text { Average peak memory used } \\
\text { (GB) }\end{array}$ & $\begin{array}{l}\text { Memory range (GB) over } \\
20 \text { replicates }\end{array}$ \\
\hline EPA-ng v.0.3.8 & $27 \mathrm{~m} \mathrm{38s}$ & $25 \mathrm{~m} \mathrm{19s}-31 \mathrm{~m} \mathrm{13s}$ & 791.82 & $781.80-800.85$ \\
\hline PAGAN2 v.1.54 & $120 \mathrm{~m} \mathrm{32s}$ & $102 m 5 s-156 m 15 s$ & 470.74 & $468.10-473.84$ \\
\hline TreeBeST v.1.9.2 & $48+h$ & N/A & $\mathrm{N} / \mathrm{A}$ & N/A \\
\hline $\begin{array}{l}\text { UShER (with preprocessed } \\
\text { mutation-annotated tree) }\end{array}$ & $0.5 \mathrm{~s}$ & $0.40-0.65 \mathrm{~s}$ & 0.28 & $0.17-0.32$ \\
\hline
\end{tabular}

A typical use case for placing SARS-CoV-2 samples onto the global phylogeny will often require placing 10-100 sequences. We did not evaluate that in this study because we found that several other algorithms could not be run on larger sample sets due to exceptionally high memory usage and runtimes. Note that while the other tools use an MSA as input, UShER accepts a VCF for new samples, which can be generated very quickly (compared to adding sequences to an existing MSA) using pairwise alignments (in, for example, minimap2 (ref. ${ }^{50}$ )) and whose overhead we ignore. We also note that

TreeBeST was not developed explicitly for this purpose; we include it in this table because it has tree placement capabilities. UShER's time and memory usage are highlighted in bold. N/A, not applicable.

(the parsimony score) required to accommodate a sample placement at a given branch. This offers an intuitive means of quantifying uncertainty in sample placements for SARS-CoV-2 phylogenies. Our placement approach and related data visualization tools are available from the University of California Santa Cruz (UCSC) SARS-CoV-2 Genome Browser $^{28}$ and will empower genomic contact tracing applications worldwide.

\section{Results}

Prior tools are inadequate for SARS-CoV-2 phylogenetics. Genomic contact tracing during this global pandemic necessitates algorithms that efficiently place samples onto the vast global tree. With this requirement in mind, we evaluated the performance of several existing approaches ${ }^{17-20}$ and compared their runtime and memory usage by adding just 1 additional sequence to a SARS-CoV-2 global phylogeny containing 38,342 leaves, our 'reference phylogeny', which comes from the 11 July 2020 release of Robert Lanfear's global SARS-CoV-2 phylogeny ${ }^{22}$. We found that the time and memory required to place a single sample is unacceptably large. For example, EPA-ng ${ }^{18}$ takes approximately 28 central processing unit $(\mathrm{CPU})$ minutes to place one sample and requires 791 gigabytes (GB) of memory (Table 1 ).

To address the challenge of real-time sample placement, we developed a new tool called Ultrafast Sample placement on Existing tRees (UShER). UShER can place a SARS-CoV-2 sample onto our reference phylogeny in just $0.5 \mathrm{~s}$, which is a $3-4$ orders of magnitude improvement over the next fastest tool (Table 1). A part of the increased efficiency of UShER stems from its heavily optimized encoding of mutations compared to a multiple sequence alignment (MSA) and from its precomputed data object storing the inferred histories of mutation events on the tree before placing samples during each execution.

UShER uses efficient tree-based data objects. Existing approaches to sample placement use an MSA of genomes that requires storing a whole-genome sequence for each sample (Fig. 1 and Methods). UShER's primary data structure is substantially more efficient. It starts with a list of variants with respect to a reference sequence for each sample and represents genotype data based on the inferred phylogeny of the viral population itself. UShER uses the FitchSankoff algorithm to infer the placement of mutations on a given tree and on the variant list ${ }^{29,30}$. Besides the phylogeny itself, UShER records only the nodes for which mutations are inferred to have occurred on the branches leading to them in a representation that we call mutation-annotated tree (Fig. 1). This representation is particularly favorable for the SARS-CoV-2 phylogeny where the mutations are relatively rare and often shared across several samples. This approach has parallels to efficient tree-based representations used recently in population genetics ${ }^{31,32}$. For our SARS-CoV-2 reference phylogeny, UShER's mutation-annotated tree uses only 3.4 megabytes $(\mathrm{MB})$ of memory, which fits easily in a last-level cache $^{33}$, to encode virtually the same information as the full MSA, which requires $1.14 \mathrm{~GB}$ (>300× improvement).

UShER can generate a mutation-annotated tree for our reference tree with 38,342 leaves and 15,129 variant positions in just 2 min $24 \mathrm{~s}$ using 4 threads (Supplementary Table 1). This data structure is then stored as a preprocessed protocol buffer (https://developers. google.com/protocol-buffers), which is a customizable binary file that can be rapidly loaded (approximately $150 \mathrm{~ms}$ ) during sample placement and data visualization (Fig. 1) and obviates the need to recompute the assignments for each execution.

Sample placement using a mutation-annotated tree. UShER uses this mutation-annotated tree to rapidly place newly acquired samples onto the tree of SARS-CoV-2 variation. More specifically, UShER uses a maximum parsimony approach where it searches the entire reference tree (Fig. 1 and Methods) for a placement that requires the fewest additional mutations to accommodate the added sample (that is, the maximum parsimony placement of a sample). UShER breaks ties based on the number of descendant leaves at the placement nodes when multiple placements are parsimony-optimal (Methods). When a preprocessed mutation-annotated tree is already available, this procedure takes approximately $0.5 \mathrm{~s}$ to place a single sample onto the SARS-CoV-2 reference tree (Table 1) and is even more efficient when placing larger sets of samples since the time to load the mutation-annotated tree is gradually reduced. For example, it only takes approximately $18 \mathrm{~s}$ to place 1,000 samples onto our reference tree using 16 threads (Supplementary Table 1). This means that our implementation is fast enough to facilitate real-time placement of SARS-CoV-2 sequences and is sufficiently memory efficient (Table 1 and Supplementary Tables 1 and 2) that everything we present could be run on a basic laptop; this should facilitate widespread adoption of this approach.

UShER accurately places simulated SARS-CoV-2 samples. To evaluate the accuracy of UShER's maximum parsimony-based placement algorithm when the viral evolutionary history is known, we generated a SARS-CoV-2-simulated dataset using a fixed tree that we supplied (Methods). UShER places samples with the correct sister node in $97.2 \%$ of cases. For samples with just 1 parsimony-optimal placement, UShER achieves $98.5 \%$ accurate local placements. When incorrect, UShER's placements tend to be quite close to the correct 
a

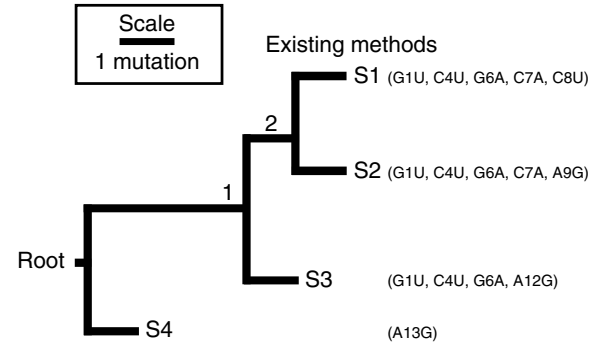

S5 (G1U, C7A)
UShER

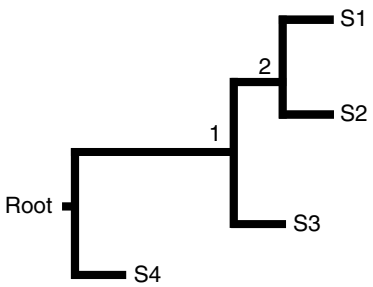

S4

\begin{tabular}{|c|c|}
\hline Node & List of mutations \\
\hline 0 (root) & $($ ) \\
\hline 1 & $(\mathrm{G} 1 \mathrm{U}, \mathrm{C} 4 \mathrm{U}, \mathrm{G} 6 \mathrm{~A})$ \\
\hline 2 & $(\mathrm{C7A})$ \\
\hline S1 & $(\mathrm{C} \mathrm{U})$ \\
\hline S2 & $(\mathrm{A} 9 \mathrm{G})$ \\
\hline S3 & $(\mathrm{A} 12 \mathrm{G})$ \\
\hline S4 & $(\mathrm{A} 13 \mathrm{G})$ \\
\hline S5 & (G1U, C7A) \\
\hline
\end{tabular}

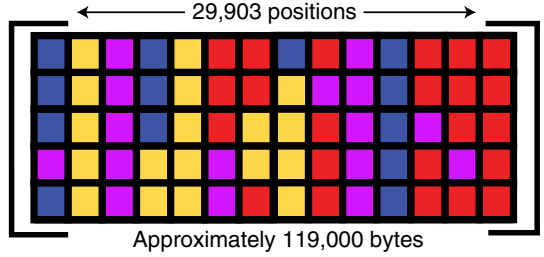

206 bytes

b

At root:

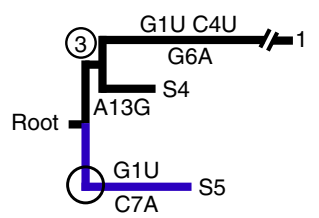

Parsimony = 2 (sibling)

At S2:

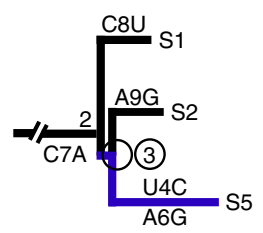

Parsimony = 2 (sibling)

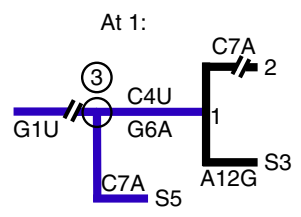

Parsimony = 1 (sibling)

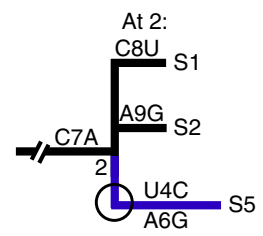

Parsimony = 2 (child)

At S3:

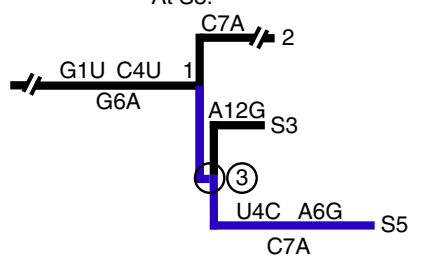

Parsimony $=3$ (sibling)

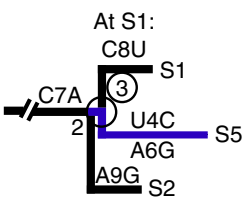

Parsimony $=2$ (sibling)

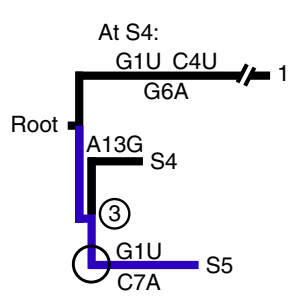

Parsimony = 2 (sibling)

c

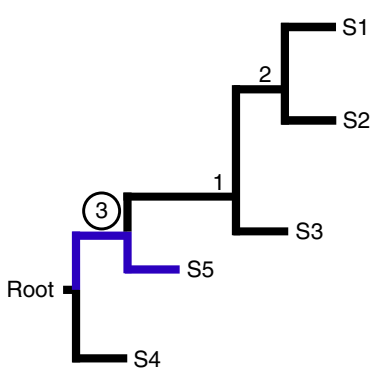

\begin{tabular}{|c|c|}
\hline Node & List of mutations \\
\hline 0 (root) & () \\
\hline 3 & $(\mathrm{G} 1 \mathrm{U})$ \\
\hline 1 & $(\mathrm{C} 4 \mathrm{U}, \mathrm{G} 6 \mathrm{~A})$ \\
\hline 2 & $(\mathrm{C7A})$ \\
\hline $\mathrm{S} 1$ & $(\mathrm{C} 8 \mathrm{U})$ \\
\hline $\mathrm{S} 2$ & $(\mathrm{~A} 9 \mathrm{G})$ \\
\hline $\mathrm{S} 3$ & $(\mathrm{~A} 12 \mathrm{G})$ \\
\hline $\mathrm{S} 5$ & $(\mathrm{C7A})$ \\
\hline S4 & $(\mathrm{A} 13 \mathrm{G})$ \\
\hline
\end{tabular}

Fig. 1 | Overview of UShER's placement algorithm and data object. a, Prior methods rely on a full MSA to inform phylogenetic structure (left), while UShER uses a mutation-annotated tree (right). The MSA shown is color-coded to match the mutations present in the tree above ( $A$, red; , yellow; $\mathrm{G}$, purple; U, blue). b, UShER evaluations of the parsimony score for placing the sample S5 (blue) at each possible position (Methods) of our example phylogeny (shown in a). We considered the branch leading to a given node to be the parent branch. The branches that need to be modified or added to the phylogeny to accommodate S5 are shown in blue; back mutations (if present) are colored red and new nodes are circled. For example, if S5 is placed at S1, the new node 3 has children S1 and S5 and two back mutations (U4C and A6G) occur at the branch leading to S5, giving this placement a parsimony score of 2. Placing S5 at node 1 is optimal by parsimony. c, The final tree with S5 added, where an additional internal node 3 is added to support S5 (left); the mutation annotations for the final tree with $\mathrm{S} 5$ colored in blue are shown on the right. Note that the memory efficiency of the mutation-annotated tree can vary depending on the dataset. In all panels, the length of each branch is proportional to the number of mutations that occurred on that branch. Zero-length branches, which are not associated with any mutations (for example, those leading to node 3 in 'at root', 'at S1', 'at S2', 'at S3' and 'at S4' in b) are shown as very short branches for visibility. 

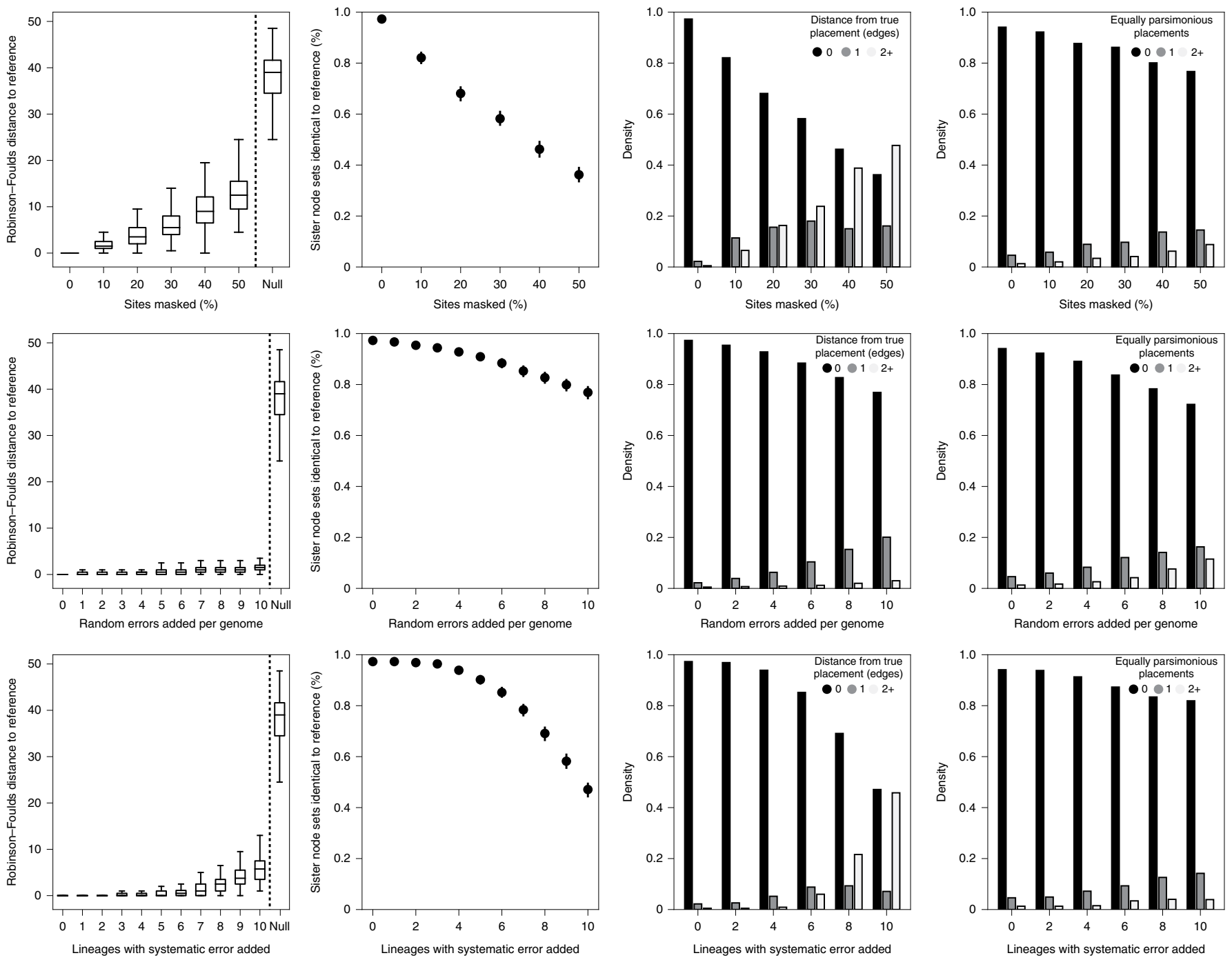

Fig. 2 | The maximum parsimony algorithm used by UShER is robust to moderate rates of missing data and simulated errors in SARS-CoV-2 genomes. Top: We independently masked sites at 10,20,30, 40 and 50 percent of sites for each of 10 simulated genomes to be added to the phylogeny and computed the Robinson-Foulds distance ${ }^{51}$, the average number of lineages added that had identical sister node sets to those in the simulated reference tree, the distance from true placement for each lineage added (Methods) and the number of equally parsimonious sites per placement for each lineage added. Middle: We introduced random nucleotide substitutions to the genomes of the 10 lineages added to the tree by UShER at a rate of 1, 2, .. 10 sites per genome, drawn independently, and computed the same measures of coherence to the reference tree, with the error bars representing the $95 \%$ confidence intervals. Bottom: We introduced one systematic error to $1,2, \ldots 10$ of the genomes added to the tree by UShER and computed the same metrics as above. For each experiment, the distance from true placement was strongly correlated with the amount of missing data $\left(P<3.34 \times 10^{-112}\right.$ for all experiments; Spearman rank correlation test with 5,998, 10,998 and 10,998 d.f. for the masking, random error and systematic error experiments, respectively). For each panel depicting RobinsonFoulds scores, the distribution of scores across 100 replicates where 10 lineages were added randomly to the phylogeny is shown to the far right for a null model comparison and is labeled 'Null'. $n=100$ replicates for each experiment. Each box plot is centered on the median of the data and extends to the first and third quartiles, with the lower whiskers extending to the lowest data point within the first quartile minus 1.5 times the interquartile range and the upper whiskers extending to the highest data point within the third quartile plus 1.5 times the interquartile range. In the error bar panels (second from the left), the data points are centered on the mean of the data and extend to the bounds of the $95 \%$ confidence interval, calculated by 1,000 iterations of bootstrapping.

node on the SARS-CoV-2 global phylogeny, that is, separated by just 1.1 edges from the correct position on the tree on average (Fig. 2 and Methods). Therefore, we conclude that UShER is capable of accurately placing new samples onto a fixed SARS-CoV-2 global reference phylogeny in practice. Although UShER works well for SARS-CoV-2, it will not be as accurate for phylogenetic analyses where maximum parsimony algorithms are known to perform poorly (for example, cases of long branch attraction ${ }^{34}$ ).

Missing data affect placement of SARS-CoV-2 genomes. Given the low mutation rate and therefore low phylogenetic signal in
SARS-CoV-2 viral genomes, missing data have a large impact on phylogenetic placement, as expected (Fig. 2). When we randomly masked between 0 and $50 \%$ of positions in samples to be placed by UShER, all measures of placement accuracy were negatively impacted. With $50 \%$ of all sites masked, only $41.9 \%$ of samples were assigned identical sister nodes as their true placement on the reference tree. However, the mean distance between UShER and correct placements on the tree remained relatively small-just 1.61 edges-and $81.0 \%$ of lineages had sister node sets in the UShER tree that were a subset of the sister nodes in the reference tree or vice versa (Methods). 
High rates of missing data have a slightly larger effect on the precision of UShER's placements than for maximum-likelihood tree inference methods when constructing a complete subtree de novo (Extended Data Fig. 1). When using Robinson-Foulds distance to measure congruence with the correct tree, we found that when no sites were masked, the average distance values from the correct tree for the trees obtained from the three methods were within $12.7 \%$ of each other and 12.9-13.3 times lower than a null model obtained from random tree construction (Extended Data Fig. 1). With no missing data, UShER produces the most congruent tree (that is, having the lowest Robinson-Foulds distance) to the correct tree, on average. The distance values increased by up to $11.1 \%$ with only $2.5 \%$ missing data and up to $76 \%$ with $10 \%$ missing data, with UShER being slightly more adversely affected by missing data than the other methods. Based on these observations, we recommend that the reference tree should ideally be maintained using only genomes with nearly complete sequences regardless of the tree inference method (for example, by filtering data obtained from the GISAID database using 'complete' and 'high coverage' tags).

UShER is robust to low error rates in SARS-CoV-2 genomes. Two types of errors in SARS-CoV-2 consensus sequences also affect the accuracy of sample placements. First, stochastic errors are likely present in many available SARS-CoV-2 sequences ${ }^{35}$. When we simulated independent errors, we found that the effects on UShER's accuracy were modest (Fig. 2). With 10 errors on average, placement is approximately $20 \%$ less likely to select the correct sister node; other distance metrics are similarly impacted (Fig. 2). Our results indicate that especially low-quality samples should be rigorously identified and excluded from analyses using UShER. Additionally, poor-quality samples can be easily flagged because they will tend to appear as unrealistically long terminal branches in UShER's placements. UShER reports all newly added samples with a parsimony score $>3$ along with a list of parsimony-increasing sites.

Second, systematic errors, where the same apparent variant is introduced into many sequences, are present in some SARS-CoV-2 sequences and have the potential to affect phylogenetic inference because they appear as inherited mutations $s^{36,37}$. Whereas UShER appears to be robust to a single systematic error present in fewer than 5 samples (Fig. 2), a single systematic error present in all 10 samples had a similar overall effect on placement accuracy as $50 \%$ missing data in error-free sequences. Consistent with our previous work $^{36,37}$, the addition of two perfectly correlated systematic errors can drastically affect UShER performance (Extended Data Fig. 2). Systematic errors should be rigorously identified and removed before sample placements are performed. We refer readers to methods that we developed previously to detect and eliminate such errors $^{36,37}$; the UShER package includes a tool to remove known problematic positions when preparing input data.

We emphasize that sequencing errors pose similar challenges for other phylogenetic inference tools (Extended Data Fig. 1) and our analysis is meant to serve as a guideline to the user rather than highlight the limitations of UShER.

Quantifying uncertainty in sample placement. Quantifying uncertainty in phylogenetic placement is critical for accurately interpreting SARS-CoV-2 phylogenies where the true phylogenetic signal is limited and sometimes even contradictor $y^{35,36}$. We developed functionality within UShER to report the number of equally parsimonious placements by default. Additionally, UShER can output the minimum number of additional mutations required to accommodate a single sample placed on each branch of the reference tree, a measure that we call the BPS. We limited this function to single sample placements because it would be challenging to quantify and represent the uncertainty imposed by the sequential incorporation of additional samples. As would be expected given the typically

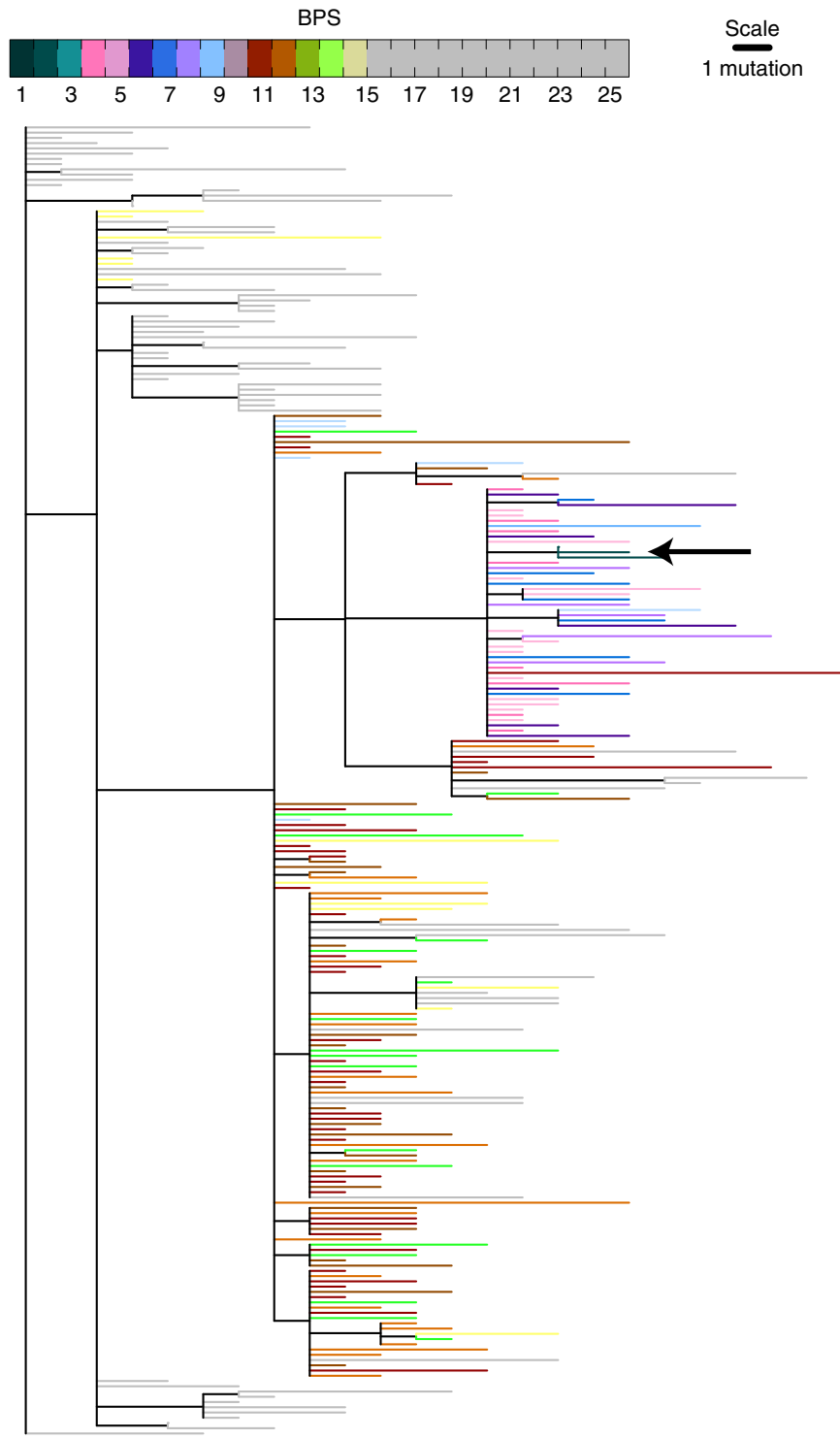

Fig. 3 | The BPS statistic for a single sample across the global SARS-CoV-2 phylogeny. The correct sample placement, which corresponds to the maximally parsimonious placement, is shown by the arrow and each branch is colored by the BPS for that sample on that branch. The phylogeny shown has been randomly subsampled to include only 250 samples for clarity of presentation. Branch lengths are measured in substitutions per genome. For the purposes of generating this illustrative figure, we placed only a single randomly selected sample $(n=1)$.

unambiguous sample placements for high-quality sequences on the global phylogeny, BPS typically increases rapidly with increasing distance along the tree (Fig. 3). Additionally, users can easily accommodate uncertainty in the input phylogeny by adding samples to many input trees in parallel. UShER can also quantify phylogenetic uncertainty within a set of samples to be placed by producing all possible topologies resulting from equally parsimonious sample placements (Extended Data Fig. 3 and Methods). To our knowledge, UShER is the only phylogenetic placement tool with this ability to produce all possible parsimony-optimal topologies and can achieve this because of its efficient data structures and high speed.

UShER is congruous with standard methods on SARS-CoV-2 data. To evaluate the performance of our approach under realistic conditions with real world SARS-CoV-2 data, we used UShER to 

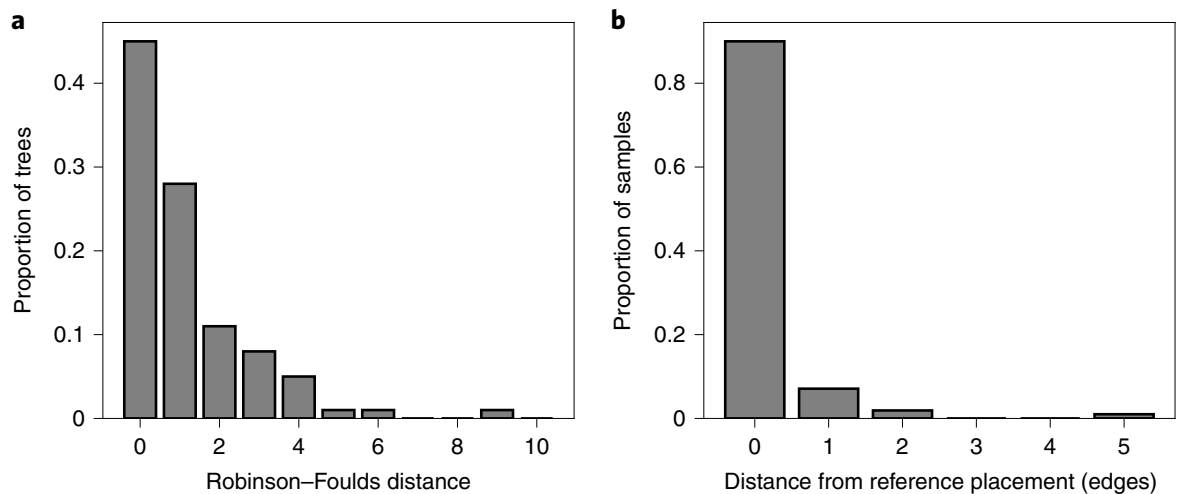

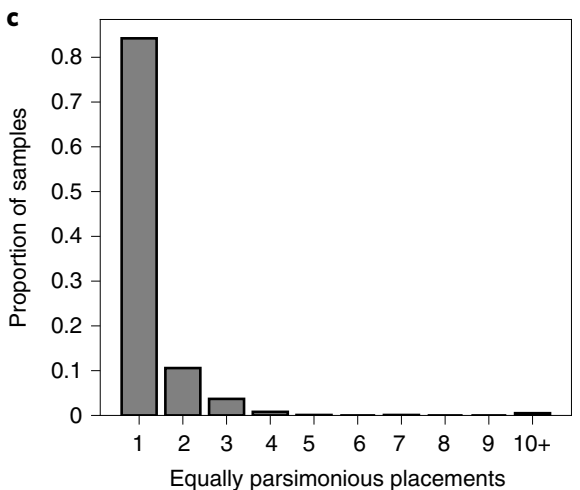

d

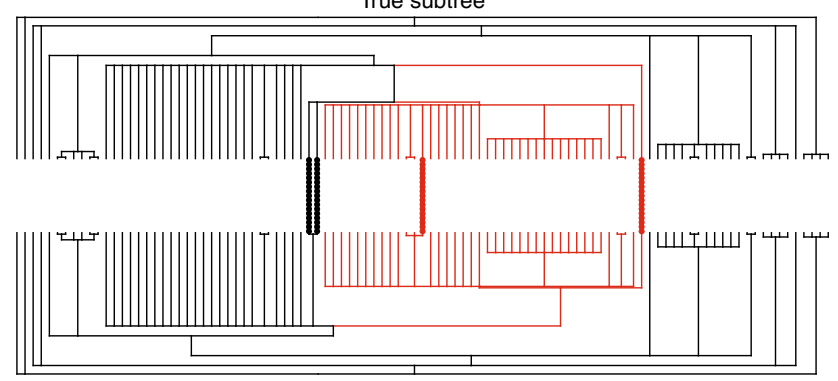

UShER constructed subtree
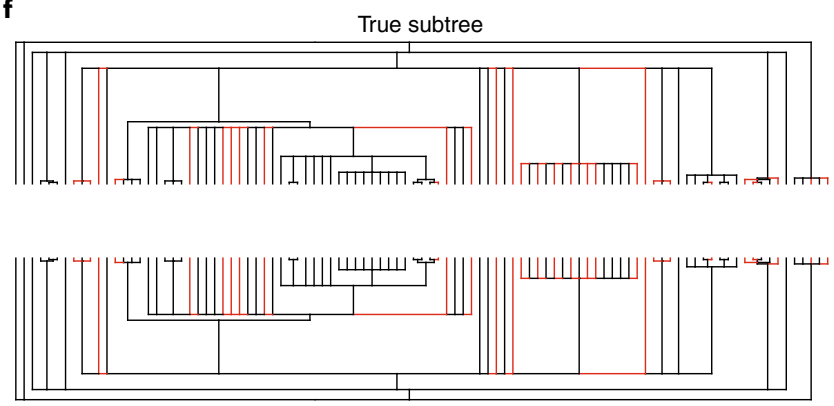

UShER constructed subtree e
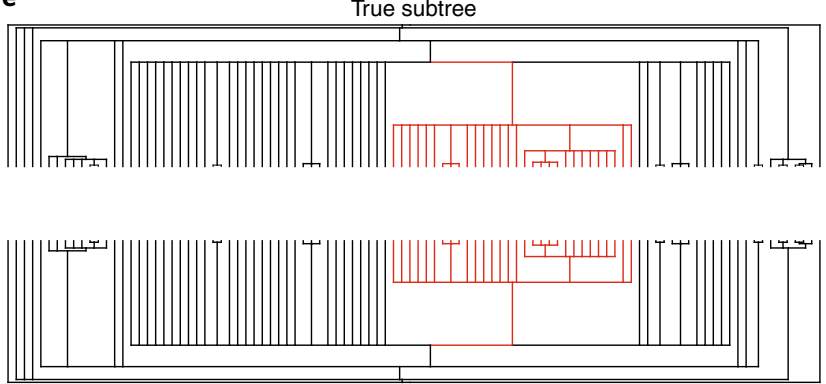

UShER constructed subtree

g
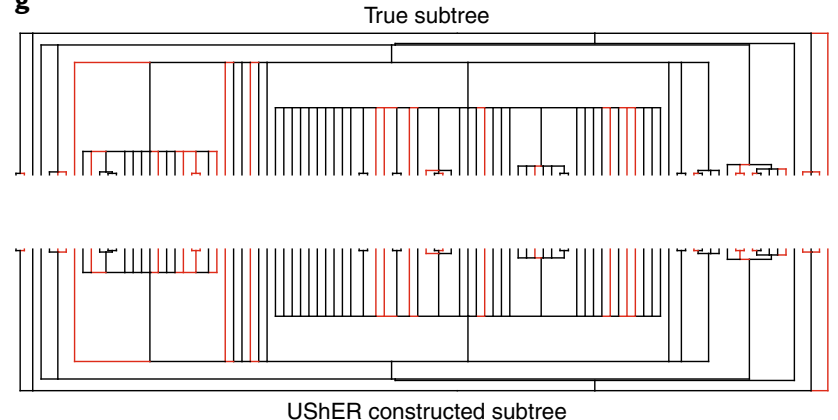

Fig. 4 | UShER is accurate using real data. a-c, Robinson-Foulds distance between 100 reference and UShER-generated trees produced by removing and re-adding 10 samples in each (a), distance from the reference placement for each of 1,000 placed samples (b) and number of equally parsimonious placements for each of the 1,000 placed samples (c) are shown. $\mathbf{d}-\mathbf{g}$, Comparisons of subsets of the global phylogeny released on 11 July 2020 with reconstruction of this phylogeny using UShER. In each case, we pruned lineages colored in red from the phylogeny and added them back using UShER. UShER accurately placed randomly selected subtrees containing lineages collected in the western United States in March and April (d) and in Europe in March (e), as well as more distantly related lineages whose times and places of collection differed more widely (f,g). $\mathbf{d}$, Differences in tree topology are highlighted in bold. $\mathbf{e}-\mathbf{g}$, Other topologies are identical. All trees in this figure are ultrametric and branch lengths are arbitrary.

place real samples onto a global reference phylogeny. Because the phylogeny was necessarily inferred from real data (Methods), this approach measures the consistency of placement between more typical tree-building approaches and the UShER placement algorithm rather than placement accuracy per se. To evaluate consistency, we randomly pruned and replaced 100 sets of 10 samples each using the reference tree (Methods). We found that UShER placed each with an identical sister node as in the reference tree in $90.0 \%$ of cases (Fig. 4). Additionally, placements tended to be quite close to correct and the mean number of edges between the reference position and UShER's placement was just 0.159 and the mean Robinson-Foulds distance for trees with 10 samples added was 1.27 (Fig. $4 \mathrm{a}-\mathrm{c}$ ). When we mimicked a plausible use case by removing larger sets of related sequences, we found that UShER can also accurately reconstruct larger subtrees for the added samples (Fig. 4d-g). Collectively, our metrics are not far from those we obtained when analyzing the simulated datasets; they indicate that missing data, errors and other features of real sequences occasionally impact UShER's placements.

We found that samples causing inconsistent placements between the reference tree and UShER were the mostly challenging cases. In particular, 6 of the 1,000 sequences that we attempted to place using UShER had large numbers of equally parsimonious placements (5-65) and were placed inconsistently relative to the reference tree. Each of these consensus sequences had a large number of ambiguous nucleotide positions (8-15) that overlapped many phylogenetically informative sites in the reference tree. This may suggest a mixture of two genetically divergent samples-either a true mixed infection or laboratory-induced. Regardless of the source, we believe future versions of the reference tree should rigorously filter sequences containing ambiguities at phylogenetically informative positions. 
Additionally increasing genetic distance and sequencing errors are expected to affect placement accuracy. We found that samples are more likely to be placed inconsistently when the parsimony score is higher $\left(P=2.98 \times 10^{-5}\right.$, one-tailed Mann-Whitney $U$-test $)$. Incorrectly placed samples also had significantly more equally parsimonious placements $\left(P=1.3 \times 10^{-21}\right.$, one-tailed Mann-Whitney $U$-test). In fact, $15 \%$ of real samples had more than 1 equally parsimonious placement on the reference phylogeny and many distinct nodes were identical in the reference tree. However, if we restricted the analysis to samples with only a single most parsimonious placement, we found that $97 \%$ of UShER's placements were consistent with the maximum-likelihood reference tree. We suggest that placement of samples that are unusually genetically distant or that have many equally parsimonious placements on a reference tree should be regarded with caution. Both statistics are reported by UShER.

UShER can maintain a global phylogeny. We propose that UShER could form the basis for 'real-time' phylogenetic platforms in periodically updating the reference tree itself or be used in conjunction with maximum-likelihood updates. To investigate this, we used UShER to add all of the 9,437 additional sequences in the 31 July 2020 release of the global tree to our 11 July 2020 reference tree. We also extensively optimized both trees using a maximum-likelihood approach in FastTree 2 (ref. ${ }^{38}$ ) (Methods and Supplementary Data 1). The Robinson-Foulds distance between all trees was similar, suggesting that the UShER updated topologies were close to de novo phylogenies (Supplementary Table 3). Additionally, the optimized version of the phylogeny produced by UShER resulted in a substantially increased likelihood over the 31 July 2020 tree inferred de novo with similarly extensive optimization (Supplementary Table 4). We obtained the highest likelihood topology from a heavily optimized 11 July 2020 tree, sample addition with UShER and then another round of tree optimization (Supplementary Table 4). This indicates that UShER, combined with additional rounds of optimization, does not result in unrecoverable local minima but rather may help avoid them. In combination with periodic maximum-likelihood updates to the global phylogeny, UShER can offer an appealing combination of real-time phylogenetic methods and model-based practices. This combination can be used to maintain an updated phylogeny for the SARS-CoV-2 pandemic.

Web interface of UShER on the UCSC SARS-CoV-2 Genome Browser. Interpretation of UShER's placements often involves scrutinizing the relationships and genotypes among closely related samples already present in the reference tree. In addition to providing the complete phylogenetic tree with new samples added, UShER can optionally provide local subtree outputs of a specified size (number of sample leaf nodes) so that the relationship of added samples to their nearest neighbors can be visualized and examined in detail. If all added samples fit within the specified size, then one subtree is created; otherwise, multiple subtrees are created as necessary to provide local subtrees for all samples.

To make genomic contact tracing using UShER widely available, we developed a Web interface integrated with the UCSC SARS-CoV-2 Genome Browser (https://genome.ucsc.edu/cgi-bin/ hgPhyloPlace). Users may upload new sample sequences in a FASTA file, or alternatively, new sample variants relative to the reference sequence (NC_045512.2/MN908947.3/Wuhan-Hu-1) in a variant call format (VCF) file. The Web server runs UShER on the new sequences and presents a summary of the sample placements to the user, with a link to download the phylogenetic tree including the newly placed sample(s). The user can click a button to view custom tracks in the Genome Browser that show subtree(s) with a configurable number of (default 50) leaves including the new sample(s) and related sequences from the initial phylogenetic tree (Extended Data Fig. 4). The Web server uses UShER's mutation-annotated tree to provide almost instant visualization. Additionally, to facilitate tree exploration and cross-referencing sequences against privately maintained personal health information, our Web interface also generates a JSON file in the Auspice v. 2 format $^{39}$ for each subtree and adds a button to view each subtree using the Nextstrain's interactive display ${ }^{40}$ (Extended Data Fig. 5). JSON files can also be downloaded and viewed behind a firewall using Auspice, which provides a drag and drop interface to view locally stored, private sample metadata (https://auspice.us ${ }^{40,41}$ ).

Widespread use of genomic contact tracing has the potential to enable public health practitioners to link apparently independent incidences of infection even across disparate sequencing centers, as well as disproving false links inferred from circumstantial evidence. This provides important and actionable information for suppressing transmission and refining public health practices. User-uploaded viral genome sequences are discarded shortly after use and not shared or stored on the UCSC Genome Browser servers unless the user saves the subtree custom tracks in a Genome Browser session. However, we stress that for global contact tracing to be maximally effective, most users must upload their viral genome sequences to public sequence repositories so that their data can also be incorporated into the reference tree. Therefore, we echo the call from the International Nucleotide Sequence Database Collaboration (https://ncbiinsights.ncbi.nlm.nih.gov/2020/08/17/ insdc-covid-data-sharing/) for all SARS-CoV-2 sequencing datasets to be made publicly available as soon as is practical.

\section{Discussion}

The SARS-CoV-2 pandemic has been accompanied by unprecedented levels of pathogen genomic sequencing, which has given rise to the opportunity for near real-time monitoring of viral transmission and evolution. This seemingly endless flood of genome sequence data has also pushed phylogenetic analysis frameworks to the extreme of their capabilities, requiring new approaches to rapidly incorporate and contextualize newly sequenced viral genomes. UShER is an extremely efficient software package inspired by a need to study the ongoing evolution of the virus itself, which provides a method to immediately incorporate viral genome isolates into a global phylogenetic tree. Compared to its closest counterpart, UShER is over 3,000 times faster, orders of magnitude more memory efficient and enables real-time genomic contact tracing. UShER is also available to the worldwide research community through a user-friendly Web interface in the SARS-CoV-2 UCSC Genome Browser. Although several challenges still remain for routinely deploying pathogen surveillance methodologies including rapid genomic data production and sharing, and outreach for public health officers, UShER removes a key barrier to pathogen surveillance by greatly decreasing the turnaround time from sample to analysis and empowering real-time genomic contact tracing efforts during the SARS-CoV-2 pandemic and beyond.

\section{Online content}

Any methods, additional references, Nature Research reporting summaries, source data, extended data, supplementary information, acknowledgements, peer review information; details of author contributions and competing interests; and statements of data and code availability are available at https://doi.org/10.1038/ s41588-021-00862-7.

Received: 30 September 2020; Accepted: 31 March 2021;

Published online: 10 May 2021

\section{References}

1. Lam, T. T.-Y. et al. Identifying SARS-CoV-2-related coronaviruses in Malayan pangolins. Nature 583, 282-285 (2020). 
2. Andersen, K. G., Rambaut, A., Lipkin, W. I., Holmes, E. C. \& Garry, R. F. The proximal origin of SARS-CoV-2. Nat. Med. 26, 450-452 (2020).

3. Zhou, P. et al. A pneumonia outbreak associated with a new coronavirus of probable bat origin. Nature 579, 270-273 (2020).

4. Shu, Y. \& McCauley, J. GISAID: global initiative on sharing all influenza data-from vision to reality. Euro Surveill. 22, 30494 (2017).

5. Stefanelli, P. et al. Whole genome and phylogenetic analysis of two SARS-CoV-2 strains isolated in Italy in January and February 2020: additional clues on multiple introductions and further circulation in Europe. Euro Surveill. 25, 2000305 (2020).

6. Surleac, M. et al. Molecular epidemiology analysis of SARS-CoV-2 strains circulating in Romania during the first months of the pandemic. Life (Basel) 10, $152(2020)$

7. Deng, X. et al. Genomic surveillance reveals multiple introductions of SARS-CoV-2 into Northern California. Science 369, 582-587 (2020).

8. Pattabiraman, C. et al. Genomic epidemiology reveals multiple introductions and spread of SARS-CoV-2 in the Indian state of Karnataka. PLoS ONE 15, e0243412 (2020)

9. Maurano, M. T. et al. Sequencing identifies multiple early introductions of SARS-CoV-2 to the New York City region. Genome Res. 30, 1781-1788 (2020).

10. Gámbaro, F. et al. Introductions and early spread of SARS-CoV-2 in France, 24 January to 23 March 2020. Euro Surveill. 25, 2001200 (2020).

11. Thielen, P. M. et al. Genomic diversity of SARS-CoV-2 during early introduction into the United States National Capital Region. Preprint at medRxiv https://doi.org/10.1101/2020.08.13.20174136 (2020).

12. Rockett, R. J. et al. Revealing COVID-19 transmission in Australia by SARS-CoV-2 genome sequencing and agent-based modeling. Nat. Med. 26, 1398-1404 (2020).

13. Dellicour, S. et al. A phylodynamic workflow to rapidly gain insights into the dispersal history and dynamics of SARS-CoV-2 lineages. Mol. Biol. Evol. 38, 1608-1613 (2021).

14. Fauver, J. R. et al. Coast-to-coast spread of SARS-CoV-2 during the early epidemic in the United States. Cell 181, 990-996.e5 (2020).

15. Lu, J. et al. Genomic epidemiology of SARS-CoV-2 in Guangdong province, China. Cell 181, 997-1003.e9 (2020).

16. Bedford, T. et al. Cryptic transmission of SARS-CoV-2 in Washington State. Science 370, 571-575 (2020).

17. Minh, B. Q. et al. IQ-TREE 2: new models and efficient methods for phylogenetic inference in the genomic era. Mol. Biol. Evol. 37, 1530-1534 (2020).

18. Barbera, P. et al. EPA-ng: massively parallel evolutionary placement of genetic sequences. Syst. Biol. 68, 365-369 (2019).

19. Löytynoja, A., Vilella, A. J. \& Goldman, N. Accurate extension of multiple sequence alignments using a phylogeny-aware graph algorithm. Bioinformatics 28, 1684-1691 (2012).

20. Ruan, J. et al. TreeFam: 2008 update. Nucleic Acids Res. 36, D735-D740 (2008)

21. Singer, J., Gifford, R., Cotten, M. \& Robertson, D. CoV-GLUE: a web application for tracking SARS-CoV-2 genomic variation. Preprint at Preprints.org https://doi.org/10.20944/preprints202006.0225.v1 (2020).
22. Lanfear, Robert. A global phylogeny of SARS-CoV-2 sequences from GISAID. Zenodo https://doi.org/10.5281/zenodo.3958883 (2020).

23. Simon, C. An evolving view of phylogenetic support. Syst. Biol. https://doi. org/10.1093/sysbio/syaa068 (2020).

24. Felsenstein, J. Confidence limits on phylogenies: an approach using the bootstrap. Evolution 39, 783-791 (1985).

25. Hoang, D. T., Chernomor, O., von Haeseler, A., Minh, B. Q. \& Vinh, L. S. UFBoot2: improving the ultrafast bootstrap approximation. Mol. Biol. Evol. 35, 518-522 (2018)

26. Minh, B. Q., Nguyen, M. A. T. \& von Haeseler, A. Ultrafast approximation for phylogenetic bootstrap. Mol. Biol. Evol. 30, 1188-1195 (2013).

27. Anisimova, M. \& Gascuel, O. Approximate likelihood-ratio test for branches: a fast, accurate, and powerful alternative. Syst. Biol. 55, 539-552 (2006).

28. Fernandes, J. D. et al. The UCSC SARS-CoV-2 Genome Browser. Nat. Genet. 52, 991-998 (2020).

29. Fitch, W. M. Toward defining the course of evolution: minimum change for a specific tree topology. Syst. Zool. 20, 406-416 (1971).

30. Sankoff, D. Minimal mutation trees of sequences. SIAM J. Appl. Math. 28, 35-42 (1975).

31. Ralph, P., Thornton, K. \& Kelleher, J. Efficiently summarizing relationships in large samples: a general duality between statistics of genealogies and genomes. Genetics 215, 779-797 (2020).

32. Kelleher, J., Thornton, K. R., Ashander, J. \& Ralph, P. L. Efficient pedigree recording for fast population genetics simulation. PLoS Comput. Biol. 14, e1006581 (2018)

33. Hennessy, J. L. \& Patterson, D. A. Computer Architecture: A Quantitative Approach (Elsevier, 2017).

34. Felsenstein, J. Cases in which parsimony or compatibility methods will be positively misleading. Syst. Zool. 27, 401-410 (1978).

35. Morel, B. et al. Phylogenetic analysis of SARS-CoV-2 data is difficult. Mol. Biol. Evol. https://doi.org/10.1093/molbev/msaa314 (2020).

36. Turakhia, Y. et al. Stability of SARS-CoV-2 phylogenies. PLoS Genet. 16, e1009175 (2020)

37. De Maio, N. et al. Issues with SARS-CoV-2 sequencing data. Preprint at https://virological.org/t/issues-with-sars-cov-2-sequencing-data/473 (2020).

38. Price, M. N., Dehal, P. S. \& Arkin, A. P. FastTree 2-approximately maximum-likelihood trees for large alignments. PLoS ONE 5, e9490 (2010).

39. Auspice v.2.0 (Nextstrain, 2020).

40. Hadfield, J. et al. Nextstrain: real-time tracking of pathogen evolution. Bioinformatics 34, 4121-4123 (2018).

41. Adding Extra Metadata via CSV/TSV-Auspice Documentation. https://docs. nextstrain.org/projects/auspice/en/latest/advanced-functionality/ drag-drop-csv-tsv.html (2020).

50. Li, H. Minimap2: pairwise alignment for nucleotide sequences. Bioinformatics 34, 3094-3100 (2018).

51. Robinson, D. F. \& Foulds, L. R. in Combinatorial Mathematics VI (eds Horadam, A. F. \& Wallis, W. D.) 119-126 (1979).

Publisher's note Springer Nature remains neutral with regard to jurisdictional claims in published maps and institutional affiliations.

(c) The Author(s), under exclusive licence to Springer Nature America, Inc. 2021 


\section{Methods}

Implementation and optimization of algorithms in UShER. Given the existing samples, whose genotypes and phylogenetic tree are known, and the genotypes of new samples, UShER aims to incorporate new samples into the phylogenetic tree while preserving the topology of existing samples and maximizing parsimony. UShER's algorithm consists of two phases: (1) the preprocessing phase; and (2) the placement phase.

In the preprocessing phase, UShER accepts the phylogenetic tree of existing samples in a Newick format and their genotypes, specified as a set of single-nucleotide variants with respect to a reference sequence (UShER currently ignores indels), in a VCF format. For each site in the VCF, UShER uses the FitchSankoff algorithm ${ }^{29,30}$ to find the most parsimonious nucleotide assignment for every node of the tree. When a sample contains ambiguous genotypes, multiple nucleotides may be most parsimonious at a node. To resolve these, UShER assigns it any one of the most parsimonious nucleotides with preference, when possible, given to the reference base. UShER also allows the VCF to specify ambiguous bases in samples using the International Union of Pure and Applied Chemistry format (https://www.bioinformatics.org/sms/iupac.html), which are also resolved to a unique base using the above strategy after inferring the most parsimonious nucleotide(s). When a branch leading to a node is found to carry a mutation, that is, the base assigned to the node differs from its parent, the mutation (for example, G6A at node 1; Fig. 1a) is added to a list of mutations corresponding to the branches leading to that node. Finally, UShER uses protocol buffers to store in a file the Newick string corresponding to the input tree and a list of lists of node mutations, which we also refer to as mutation-annotated tree object, as shown in Fig. 1. The outer list is ordered according to the depth-first traversal of nodes. UShER also parallelizes the independent Fitch-Sankoff computations for multiple VCF sites efficiently using multiple threads (Supplementary Tables 1 and 2).

In the placement phase, UShER loads the preprocessed mutation-annotated tree and the genotypes of new samples in a VCF format and sequentially adds the new samples to the tree. For each new sample, UShER computes the additional parsimony score required for placing it at every node in the current tree while considering the full path of mutations on the branches from the root of the tree to that node (Fig. 1b). For internal nodes, the parsimonious placement can be as a sibling to the node (for example, node 1 in Fig. 1b), when there are mutations on the branch leading to that node that are not shared by the new sample or as a child to that node (for example, node 2 in Fig. 1b), when all mutations on the branch leading to that node are shared by the new sample. For leaf nodes, only sibling placement is considered (Extended Data Figs. 1-4 and Fig. 1b) to ensure that samples are always maintained as leaves of the tree. Next, UShER places the new sample at the node that results in the smallest additional parsimony score. While placing a new sample, UShER parallelizes the parsimony score computation over different nodes of the tree using multiple threads.

When multiple node placements are equally parsimonious, UShER uses the following tie-breaking strategy that has good empirical results (based on simulated data). When comparing two independent equally parsimonious placements, UShER picks the node with a greater number of descendant leaves for placement. However, if the choice is between a parent and its child node, UShER picks the parent node if the number of descendant leaves of the parent that are not shared with the child node exceed the number of descendant leaves of the child. We note that maximum likelihood might perform better in tie-breaking between otherwise equivalent placements in some applications; however, the SARS-CoV-2 maximum-likelihood approaches appear to perform similarly to UShER's strategy described above, suggesting that this is not a key challenge for our application (Extended Data Fig. 1). To limit the uncertainty in sample placement that often arises out of incomplete sequences, UShER also provides an option to specify the maximum number of equally parsimonious placements allowed for placing a sample. The default value is set high such that a placement is always carried out by default.

At the end of the placement phase, UShER allows the user to create another protocol buffer file containing the mutation-annotated tree object for the newly generated tree including added samples (Fig. 1c). This allows another round of placements to be carried out over and above the newly added samples. While UShER's sequential placement of new samples helps it achieve high speed, the placements could potentially be worse than a full de novo tree inference procedure. However, in practice, we have found UShER's accuracy over iterated placements to be reasonably high.

UShER implements a few additional optimizations to speed up the placement phase. For example, the parsimony score for a node requires computing the symmetric set difference between the set of new sample variants and the set of mutations on the branches from the root of the tree to that node. UShER maintains mutations sorted by positions to speed up this computation. UShER also maintains the minimum parsimony score of previously traversed nodes in a shared variable and terminates the computation of the set difference in a new node as soon as the parsimony score corresponding to it exceeds the value of this shared variable. Finally, UShER also allows an option during the preprocessing phase to collapse nodes (that is, delete the node after moving its child nodes to its parent node), branches leading to which are not inferred to contain a mutation through the Fitch-Sankoff algorithm as well as condensing nodes in a polytomy that contains identical sequences into a single representative node, both of which help in greatly reducing the search space for the placement phase.

Branch lengths in UShER. Since UShER is based on maximum parsimony, by default it reports branch length as the number of mutations assigned to that branch. However, UShER also provides an option to the user to retain the original branch lengths from the input tree, which could be, for example, maximum-likelihood estimates for substitutions per site, for all branches that are unaffected by sample placement, while branch lengths for new branches and those modified during the placement are undefined (that is, they do not have an associated value in the Newick format). We do this to avoid inconsistencies between branch lengths in the resulting augmented trees.

Enumerating all possible parsimony-optimal placement topologies in UShER. To further aid the user to quantify phylogenetic uncertainty in placement, UShER provides an option to enumerate all possible topologies resulting from equally parsimonious sample placements. UShER does this by maintaining a list of mutation-annotated trees, starting with a single mutation-annotated tree corresponding to the input tree of the existing samples, and sequentially adds new samples to each tree in the list while increasing the size of the list as needed to accommodate multiple equally parsimonious placements for a new sample. To keep the runtime and memory usage of UShER reasonable, the user specifies the maximum number of topologies that UShER should maintain before it reverts back to using the default tie-breaking strategy described above to pick a single placement among multiple equally parsimonious placements for the subsequent samples, therefore avoiding a further increase of the list size. Note that if the number of equally parsimonious placements for the initial samples is large, the tree space can get large too quickly and slow down the placement for the subsequent samples. Therefore, UShER also provides an option to sort the samples first based on the number of equally parsimonious placements.

Simulating realistic SARS-CoV-2 genome evolution. To evaluate the accuracy of our placement algorithm on a known phylogeny, we produced a set of simulated samples for which we knew the correct placement on the tree. To do this, we first needed to obtain a mutation rate matrix. Each position of the reference genome (NC_045512.2/MN908947.3/Wuhan-Hu-1; https://www.ncbi.nlm.nih. gov/nuccore/MN908947) was classified as coding or noncoding. Start and stop codons were not considered and were simulated as constant. The first and last 100 base pairs of the genome and sites marked as problematic in https://github. com/W-L/ProblematicSites_SARS-CoV2/blob/master/problematic_sites_sarsCov2. $\mathrm{vcf}$ were also not considered for estimating substitution rates but they were simulated like the other 'normal' sites. We counted 'opportunities' of mutations based on the reference genome: for example, a noncoding $\mathrm{C}$ allele in the reference genome represents three opportunities for noncoding mutations $(C>A, C>G$ and $\mathrm{C}>\mathrm{U}$ ). For coding sites, we split synonymous and nonsynonymous mutation opportunities into two separate counts. Then, we counted the number of observed mutation events of each type using the inferred mutational history of the viral population based on the phylogeny and global alignment from 11 July 2020 and using the software we described previously (https://github.com/yatisht/ strain_phylogenetics ${ }^{37}$ ). As above, we masked the ends of the genome, previously identified problematic sites and start/stop codons.

To further avoid potential bias resulting from sequencing errors, RNA degradation, contamination or other possible sources of error, we used a threshold for minor allele counts for variants/mutations to be included in our analysis. For all the results presented, we used rate estimates obtained including only variants/ mutations with at least a minor allele count of 7 . Results varied a little by varying this threshold; for example, the nonsynonymous/synonymous substitution rate ratio $\omega$ usually tended to decrease with increasing this threshold $(\omega=0.48$ with threshold $3, \omega=0.48$ with threshold 7 and $\omega=0.43$ with threshold 20). However, overall, the effect is limited (Supplementary Tables 5-10). Also, we used two different ways to count mutation events. In the first approach, we used the reconstructed mutation histories from the strain_phylogenetics package (commit eb978ac) and only counted substitution events with at least seven descendant lineages. The other approach only used the alignment and ignored the reconstructed mutation history: we counted variant alleles in the alignment that had a minor allele count of at least seven. These two approaches give comparable results (Supplementary Tables 5-10); in this study, we used the results from the first approach

For each class of mutations (synonymous or nonsynonymous) and from any nucleotide to any other nucleotide, we calculated a rate by dividing its total opportunity count by its mutation count. Given the small number of noncoding sites, we merged the noncoding and synonymous counts.

Simulating sequence evolution along the tree. We assumed a root genome equal to the reference genome and simulated its evolution along a phylogeny with 38,342 leaves using pyvolve v.1.0.1 (ref. ${ }^{42}$ ). We simulated the evolution of each site with a nucleotide substitution process while still taking into account the genetic code and a non-neutral nonsynonymous/synonymous rate ratio $(\omega)$ in doing so. We achieved this by assuming that the codon context of each position was constant; 
this is sufficiently realistic because it is very rare that two positions in the same codon are both mutated (with respect to the reference) in the same SARS-CoV-2 sequence. So, considering each genome position in turn, we evolved noncoding sites under the synonymous matrix above; for coding sites, we used the same rates for synonymous mutations, while we multiplied the rate of nonsynonymous mutations by $\omega$. The reason for using this approach instead of simulating under a codon model is that a codon model would have been prohibitively computationally intensive.

We assumed that $\omega$ was variable across sites and tested the resilience of our approach to variation in rates across sites. We chose a distribution of $\omega$ such that the average simulated $\omega$ was close to the $\omega$ inferred from real data: $20 \%$ probability of $\omega=0.01 ; 25 \%$ probability of $\omega=0.1 ; 25 \%$ probability of $\omega=0.4$; and $30 \%$ probability of $\omega=1$. Pyvolve automatically rescaled the input rate matrices, so we rescaled the tree at each site so that the synonymous rates were the same across sites. In addition, we rescaled the overall tree so that the observed number of mutation events in the simulated alignment was similar to the number in the real alignment.

Accuracy evaluation. We measured UShER's accuracy in placing samples onto a reference phylogeny using simulated (described above) and real data. For simulated data, both reference phylogeny and sequences were simulated; for real data, we used the global phylogeny dated 11 July 2020 (https://github.com/ roblanf/sarscov2phylo; Supplementary Data 1) as reference and its corresponding sequences were obtained from GISAID ${ }^{4}$. In each case, we first randomly pruned out ten samples from the global phylogeny, which was then used as the input phylogeny while adding back the pruned samples using UShER. UShER's accuracy in placing back the samples was computed using the average values of three different statistics (described below) over 100 such replicates.

We initially used TreeCmp ${ }^{43}$ v.2.0-b76 to compute the Robinson-Foulds distance between the reference phylogeny and the tree constructed by samples using UShER. Separately, we recorded whether the sister clade for each placed sample was identical to the true sister clade (that is, the sister clade in the reference phylogeny). Finally, we computed the distance between the UShER placement and the correct placement in terms of the minimum number of edges separating them as described below.

Ordinarily, the distance between two nodes in a tree can be computed using their lowest common ancestor ${ }^{44}$ by taking the sum of the number of edges to each node from the lowest common ancestor. To determine the distance between the node placement in two trees (reference phylogeny and the one resulting from UShER placement), we developed a utility that reports all descendant lineages from an $n$-th generation ancestor of any given node in a tree, with $n$ provided as input (that is, when $n=1$, it reports unpruned lineages in the sister clade). For each pruned lineage, we found the descendants varying the number of generations, N1 and N2, in global and UShER phylogenies, respectively and reported the distance between the UShER placement and the correct placement as the smallest $(\mathrm{N} 1+\mathrm{N} 2$ -2 ), which resulted in the same set of descendant lineages in both phylogenies. Note that the second statistic records cases for which the sister clades in the two trees are identical, which would always have 0 distance in our third statistic $(\mathrm{N} 1=\mathrm{N} 2=1$; Extended Data Fig. 6).

We also measured UShER's accuracy in a more realistic scenario of placing closely related samples that form their own subtree. In this case, during pruning, we required that the pruned samples together formed a subtree (that is, not a trivial polytomy) in the reference phylogeny.

To evaluate the accuracy and robustness to error of UShER compared to IQ-TREE 2 and FastTree 2, we identified 5 clades of approximately 1,000 lineages and reconstructed each from scratch using each of the 3 methods, and repeated these experiments after randomly masking between 2.5 and $50 \%$ of sites to ' $n$ ', adding 10, $20 \ldots 100$ independently drawn random single-nucleotide errors across the lineages to be placed and adding identical single-nucleotide errors to 1-10 of the genomes to be placed (Extended Data Fig. 1). We measured the accuracy of these placements by calculating the Robinson-Foulds distance using TreeCmp ${ }^{43}$ v2.0-b76 (Extended Data Fig. 6).

Benchmarking placement algorithms. We compared UShER to four other lineage placement algorithms: IQ-TREE multicore v.2.1.1; EPA-ng v.0.3.8; PAGAN2 v.1.54; and TreeBEsT v.1.9.2 (refs. ${ }^{17-20}$ ). We initially attempted to add 1,000 lineages to our simulated phylogeny; however, except or UShER, which required $18 \mathrm{~s}$ to finish using 64 threads, none of the placement programs finished within $24 \mathrm{~h}$. Due to time and memory constraints, we instead added only 1 lineage to the tree in 20 replicates, recording the average and time range and peak memory usage across these 20 replicates in Table 1. A full list of commands used to run each test can be found in Supplementary Table 11. We installed and ran each program on a server with 160 processors (Intel Xeon CPU E7-8870 v.4, 2.10 gigahertz), each with $20 \mathrm{CPU}$ cores.

Tree construction for SARS-CoV-2 samples. Full details and reproducible code for the construction of the global tree of SARS-CoV-2 samples are available in the 31 July 2020 release and at Lanfear ${ }^{22}$. To summarize, this code creates a global phylogeny of all available samples from the GISAID data repository as follows.
First, all sequences marked as 'complete' and 'high coverage' submitted up to 31 July 2020 were downloaded from GISAID. Sequences with known issues from previous analyses were then removed from this database (details are in the excluded_sequences.tsv file; https://github.com/roblanf/sarscov2phylo/blob/master/ excluded_sequences.tsv). Second, a global alignment was created by aligning every sequence individually to the NC_045512.2 accession from the National Center for Biotechnology Information using mafft v.7.471 (ref. ${ }^{45}$ ), faSplit (http://hgdownload. soe.ucsc.edu/admin/exe/), faSomeRecords (https://github.com/ENCODE-DCC/ kentUtils), both version v.377, and GNU Parallel v.20200822 $2^{46}$. This approach aligns each sequence individually to the reference, then joins them into a global alignment by ignoring insertions relative to the reference. Third, sites that are likely to be dominated by sequencing errors ${ }^{36}$ are masked from the alignment using faSplit, seqmagick v.0.7.0+7.g1642bb8 (https://seqmagick.readthedocs.io/en/ latest/) and GNU Parallel; sequences shorter than 28 kilobases and/or with $>1,000$ ambiguities are removed from the alignment using esl-alimanip v.0.47 (http:// www.hmmer.org/); subsequently, sites that have $>50 \%$ gaps are removed (after converting $N$ to gaps) with esl-alimask. Fourth, the global phylogeny is estimated using Fast Tree 2 (ref. ${ }^{38}$ ) (v.2.1.10 compiled with double precision) in two stages: (1) an initial analysis that produces a neighbor joining tree, which is optimized with 5 rounds of subtree pruning and regrafting (SPR) moves of length 500; and (2) a second analysis, which uses the tree from the first analysis as a starting tree with 5 rounds of SPR moves of length 200 and otherwise default FastTree 2 settings. Finally, goalign (commit v.0.3.1; https://github.com/evolbioinfo/goalign) was used to create 100 bootstrap alignments followed by reestimating all the maximum-likelihood trees with FastTree 2 with the -fastest setting, using GNU Parallel to manage parallelization. The resulting trees were rooted with our reference (NC_045512.2/MN908947.3/Wuhan-Hu-1) sequence using nw_reroot v.1.6 (ref. ${ }^{47}$ ).

From the resulting tree, we removed sequences on very long branches using TreeShrink v.1.3.7 (refs. ${ }^{48,49}$ ) using the default $q \leq 0.05$ threshold to identify such branches. These sequences are likely to be either of poor quality and/or poorly aligned, so rather unreliable to interpret in a phylogeny with such limited variation.

Tree optimization for inferred SARS-CoV-2 phylogenies. For the 11 and 31 July 2020 reference trees, we created 'optimized' versions of each using FastTree 2 (ref. ${ }^{38}$ ) using ten iterations of the command 'fasttree -nt -nni 0 -spr 1 -sprlength 1000 -nosupport -intree $<$ initial tree $>$ global.fa $><$ new tree $>$ ', replacing the initial tree with the new tree from the previous iteration each time, followed by the command 'fasttree -nt -nni 0 -spr 1 -sprlength 1000 -nosupport -gamma -intree <initial tree $>$ global.fa $><$ new tree $>$ '. In these commands, -nt indicates that the input is a nucleotide alignment, -nni 0 indicates that no minimum-evolution nearest neighbor interchanges are done, -spr 1 -sprlength 1000 indicates 1 round of SPR with a maximum distance of 1,000 , meaning that a single SPR move could move a subtree to any new branch of the global tree. The -nosupport flag indicates that support values are not output and the -gamma flag indicates that the lengths are rescaled to optimize the Gamma20 likelihood ${ }^{38}$. Because FastTree 2 requires binary trees, we randomly resolved all polytomies before optimization. We also generated two other trees using UShER, by taking the original and 'optimized' 11 July trees, pruning out all lineages in the 11 July 2020 tree not present in the 31 July 2020 tree and using UShER to add in all lineages present in the 31 July 2020 that were not present in the 11 July tree. We then optimized these two new trees using ten iterations of FastTree 2, followed by another round of optimization using the -gamma flag as described above.

Parsing the mutation-annotated tree for subtree visualization. When we were developing the Web application for UShER, we discovered that parsing genotype data from a VCF file containing 40,000+ samples was the most time-consuming step for displaying the resulting subtrees. Therefore, we developed an approach for parsing genotype data from subtrees from the mutation-annotated tree object. Briefly, our approach descends from the root of the phylogeny to the focal subtree, accumulates the relevant mutations along the path and then extracts the variation within the subtree that will be used for visualization. This heavily reduced dataset can then be visualized using the existing code base of the UCSC Genome Browser, outputting a JSON-formatted file that can be viewed using Auspice (https:// nextstrain.github.io/auspice/). With the current dataset sizes, this procedure takes approximately $0.03 \mathrm{~s}$ in total to extract genotype data for a subtree of 50 sequences. Software for rapid subtree VCF extraction from our mutation-annotated tree object is available at https://github.com/ucscGenomeBrowser/kent/tree/master/src/hg/ hgPhyloPlace/phyloPlace.c.

Reporting Summary. Further information on research design is available in the Nature Research Reporting Summary linked to this article.

\section{Data availability}

All data used in this work are available from GISAID (https://www.gisaid.org/), with specific sample accessions listed in Supplementary Data 2-6.

\section{Code availability}

UShER is available to users through the UCSC Genome Browser at https://genome. ucsc.edu/cgi-bin/hgPhyloPlace. The source code and detailed instructions on 
how to compile and run UShER are available at https://github.com/yatisht/usher. The code used for the statistical analyses and to produce the figures is available at https://github.com/bpt26/USHER_ANALYSES.

\section{References}

42. Spielman, S. J. \& Wilke, C. O. Pyvolve: a flexible Python module for simulating sequences along phylogenies. PLoS ONE 10, e0139047 (2015).

43. Bogdanowicz, D., Giaro, K. \& Wróbel, B. TreeCmp: comparison of trees in polynomial time. Evol. Bioinform. Online 8, 475-487 (2012).

44. Bender, M. A., Farach-Colton, M., Pemmasani, G., Skiena, S. \& Sumazin, P. Lowest common ancestors in trees and directed acyclic graphs. J. Algorithms 57, 75-94 (2005)

45. Katoh, K. \& Standley, D. M. MAFFT multiple sequence alignment software version 7: improvements in performance and usability. Mol. Biol. Evol. 30, 772-780 (2013).

46. Tange, O. GNU Parallel: the command-line power tool. USENIX Mag. https://www.usenix.org/publications/login/february-2011-volume36-number-1/gnu-parallel-command-line-power-tool (2011).

47. Junier, T. \& Zdobnov, E. M. The Newick utilities: high-throughput phylogenetic tree processing in the Unix shell. Bioinformatics 26, 1669-1670 (2010).

48. Mai, U. \& Mirarab, S. TreeShrink: fast and accurate detection of outlier long branches in collections of phylogenetic trees. BMC Genomics 19, 272 (2018)

49. Paradis, E. \& Schliep, K. ape 5.0: an environment for modern phylogenetics and evolutionary analyses in R. Bioinformatics 35, 526-528 (2019).

52. Bouckaert, R. R. DensiTree: making sense of sets of phylogenetic trees. Bioinformatics 26, 1372-1373 (2010).

\section{Acknowledgements}

We thank all of the Nextstrain team members whose platform we have built on as a part of this work. We also thank J. Batson, S. Bell and M. Bui for providing feedback on the UShER features. Until about the 24 June 2020, GISAID acknowledgements were provided in tabulated form on their website; therefore, they could be combined into a single file. This is Supplementary Data 2. Subsequent to this, GISAID shifted to only allowing PDF downloads of this table. However, it is not possible to download the entire table at once, only small subsections of it. As a result, acknowledgements after the 24 June 2020 are provided in PDF format (Supplementary Data 3-6). This statement applies to all formats of the acknowledgement tables. We thank the following authors from the originating laboratories responsible for obtaining the specimens and the submitting laboratories where genome data were generated and shared via GISAID, on which this research is based. During this work, Y.T is funded through Schmidt Futures Foundation SF 857 and NIH grant 5R01HG010485. B.T. and R.C.-D. were supported by grant no. R35GM128932 and by an Alfred P. Sloan Foundation Fellowship to R.C.-D. B.T. was funded by grant nos. T32HG008345 and F31HG010584. The UCSC Human Genome Browser software, quality control, and training is funded by National Human Genome Research Institute, currently with grant no. 5U41HG002371-19. The SARS-CoV-2 genome browser and data annotation tracks are funded by generous individual donors including P. and R. Rebele, E. and W. Schmidt by recommendation of the Schmidt Futures program, the Center for Information Technology Research in the Interest of Society (no. 2020-0000000020) and a University of California Office of the President Emergency COVID-19 Research Seed Funding Grant no. R00RG2456. N.D.M. is funded by the European Molecular Biology Laboratory. R.L. is funded by an Australian Research Council grant no. DP200103151 and by a Chan Zuckerberg Initiative grant.

\section{Author contributions}

R.C.-D. and Y.T. conceived and designed the research. N.D.M., B.T., A.S.H., N.D.M., L.G. and R.L. performed the research and analyses. Y.T., A.S.H. and L.G. developed the software tools. D.H. and R.C.-D. contributed reagents and resources. R.C.-D., Y.T. and B.T. wrote the manuscript with input from all authors. All authors edited and contributed to the manuscript revision.

\section{Competing interests}

A.S.H. and D.H. receive royalties from the sale of UCSC Genome Browser source code licenses to commercial entities. R.L. works as an advisor to GISAID. The remaining authors declare no competing interests.

\section{Additional information}

Extended data is available for this paper at https://doi.org/10.1038/s41588-021-00862-7.

Supplementary information The online version contains supplementary material available at https://doi.org/10.1038/s41588-021-00862-7.

Correspondence and requests for materials should be addressed to Y.T. or R.C.-D.

Peer review information Nature Genetics thanks Matthew Hall and Art Poon for their contribution to the peer review of this work. Peer reviewer reports are available.

Reprints and permissions information is available at www.nature.com/reprints. 

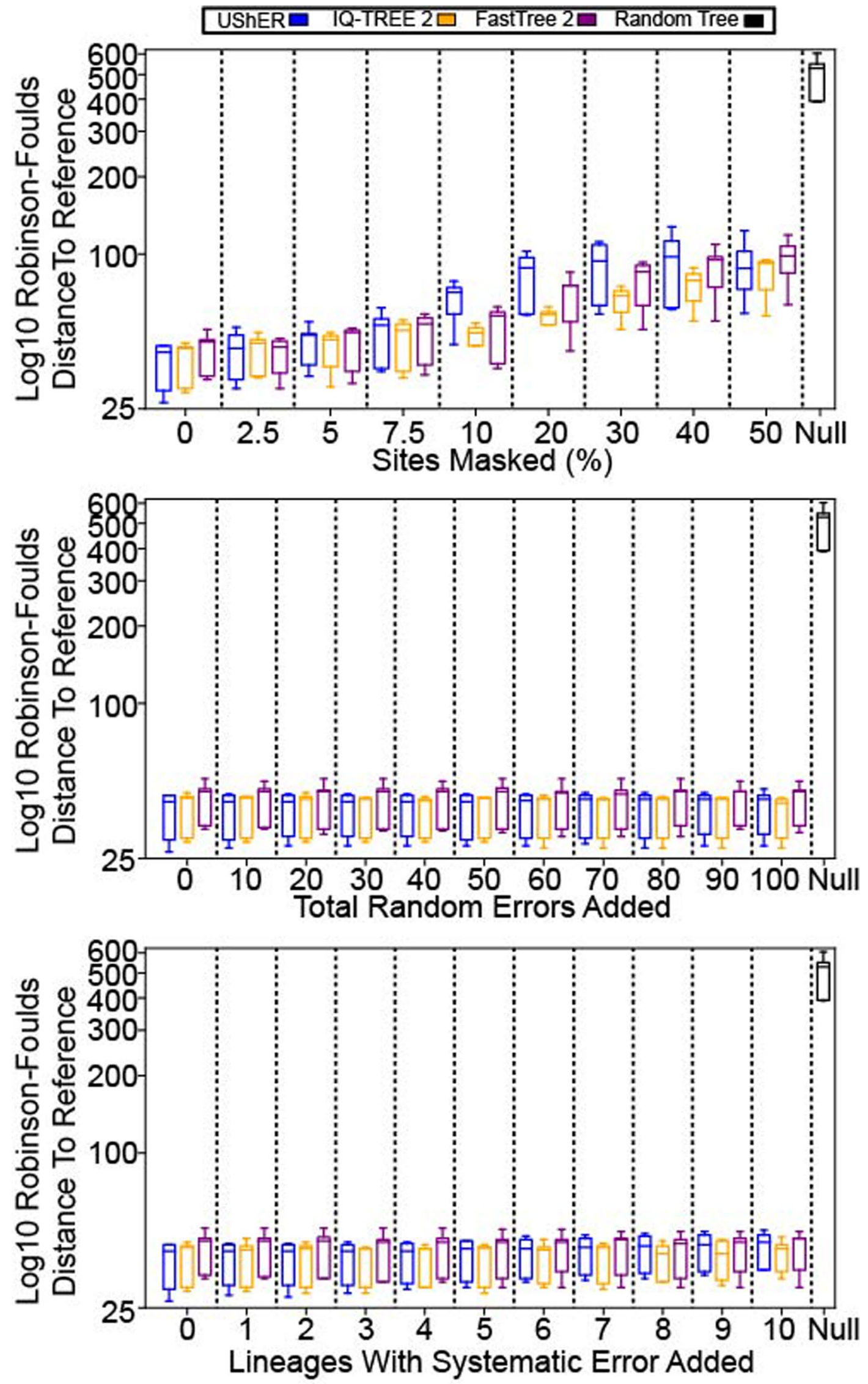

Extended Data Fig. 1 | See next page for caption. 
Extended Data Fig. 1 | UShER is similarly robust to masked sites and nucleotide errors as IQ-TREE2 and FastTree 2. We pruned 5 independent clades of roughly 1,000 lineages each and applied the same methods as in Fig. 2, masking 2.5, 5, 7.5, 10, 20 ...50 percent of sites (top, note that the X-axis does not use a linear scale), adding 10, 20...100 independently drawn random nucleotide substitutions across the lineages to be placed (center), and adding one error to 1, 2...10 of the genomes of interest (bottom). We then used UShER (blue), IQ-TREE $2^{17}$ (orange), and FastTree $2^{38}$ (purple) to reconstruct these clades. We determined the Robinson-Foulds distance of each to the original clade using TreeCmp ${ }^{43}$, as well as the distance of randomly constructed trees to the far right (black, labeled 'Null') as a null model comparison. $\mathrm{N}=5$ independent replicates for each experiment. Each boxplot is centered on the median of the data and extends to the first and third quartiles, with whiskers extending to the minimum and maximum of the data set. 
ARTICLES

NATURE GENETICS
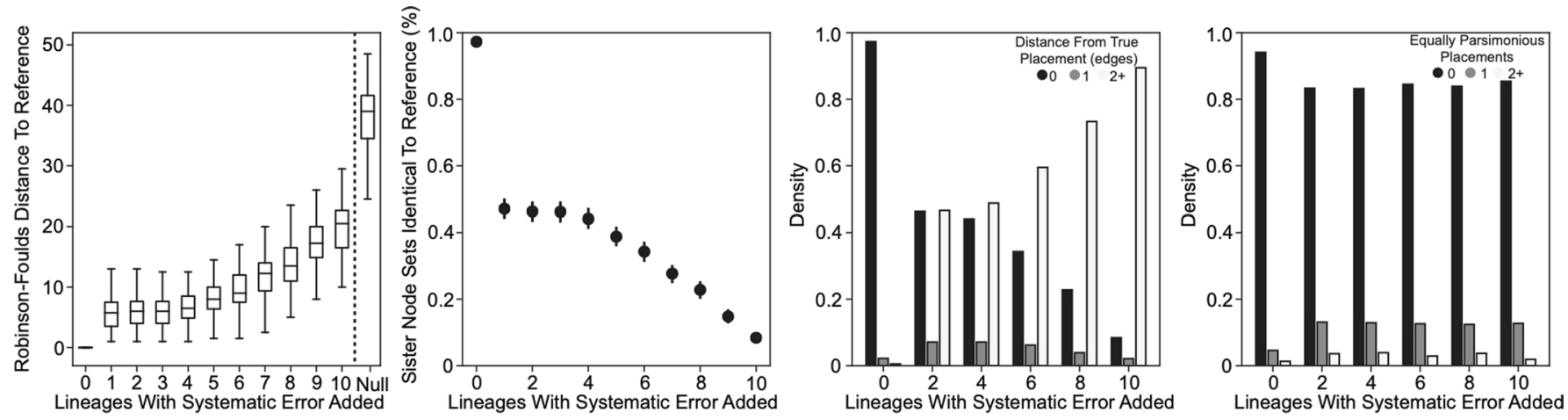

Extended Data Fig. 2 | Addition of two perfectly correlated errors significantly reduces UShER accuracy. As in Fig. 2, the Robinson-Foulds distances, proportion of sister nodes identical to the reference tree, distance from true placement and equally parsimonious placements, respecitvely, are shown for UShER experiments in placing 10 lineages, with two perfectly correlated errors added to $1,2 \ldots 10$ of the lineages to be placed. To the far right in the left-most panel, labeled 'Null', the distribution of scores across 100 replicates in which 10 lineages were added randomly to the phylogeny is shown as a null model for comparison. $\mathrm{N}=100$ independent replicates for each experiment. The whiskers in the boxplot on the left are centered on the median of the data and extend to the first and third quartiles. In the error bars panel (second from the left), the data points are centered on the mean of the data and extend to the bounds of the $95 \%$ confidence interval, calculated by 1,000 iterations of bootstrapping.

NATURE GENETICS | www.nature.com/naturegenetics 

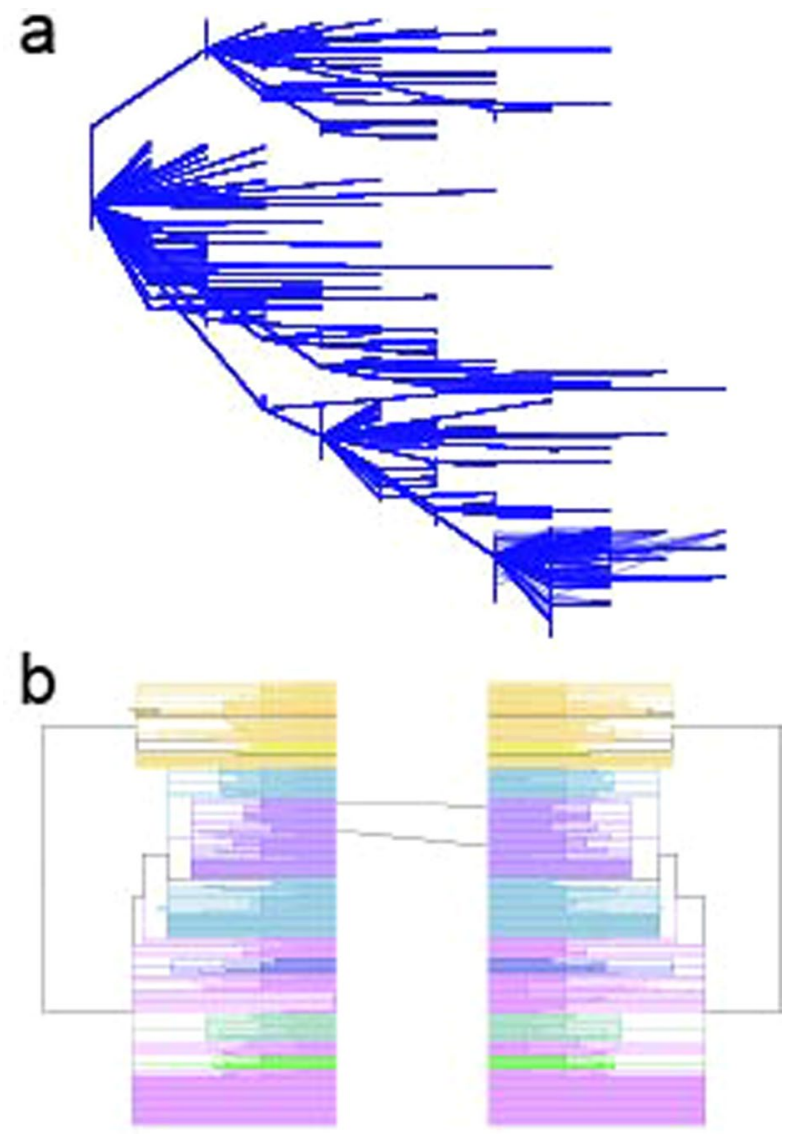

Extended Data Fig. 3 | UShER can output multiple trees to accommodate phylogenetic uncertainty. (A): Composite of 239 trees with 424 samples, representing all possible parsimony-optimal placements of two samples on a starting tree having 422 samples, computed using DensiTree ${ }^{52}$ and plotted using the phangorn package (https://cran.r-project.org/web/packages/phangorn). All trees were scaled to be the same height. (B): Two of the trees from (A) compared in a tanglegram, colored according to COG-UK lineage assignments, with linker lines shown only for the two placed samples whose placements differ between topologies. As in Fig. 4, both trees in this tanglegram are ultrametric and branch lengths are arbitrary. 


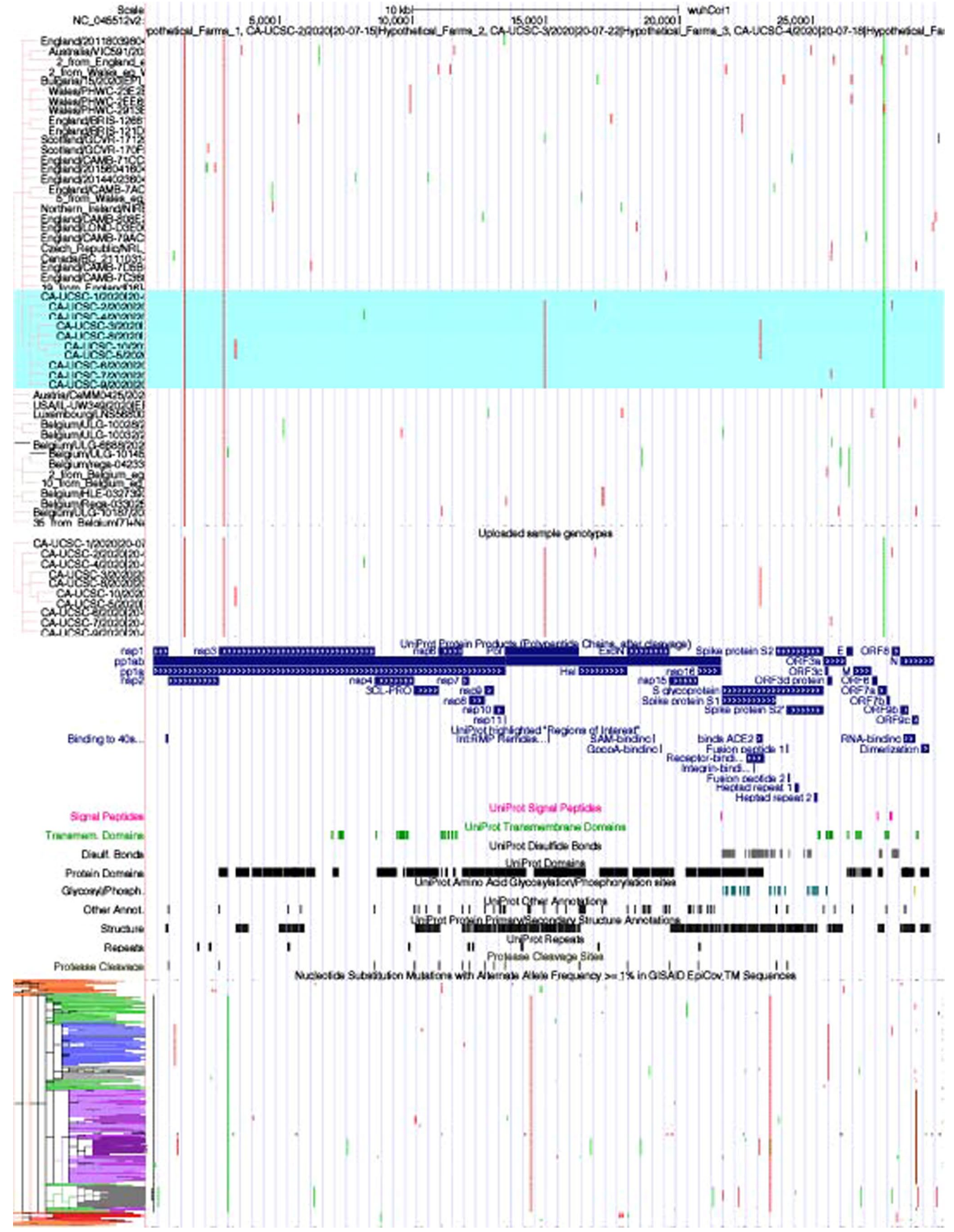

Extended Data Fig. 4 | See next page for caption. 


\section{NATURE GENETICS}

Extended Data Fig. 4 | UCSC Genome Browser display of subtree where hypothetical example sequences have been placed by UShER. Newly added samples are highlighted in blue and the tree displaying their relationships and placement on the global tree is shown to the left. Interactive view: https:// genome.ucsc.edu/s/AngieHinrichs/UShER_example. 
Sample typev

uploaded sample
GISAID sample

\section{C241T|G1440A|G2891A|T241C|C28887T|A27669G}

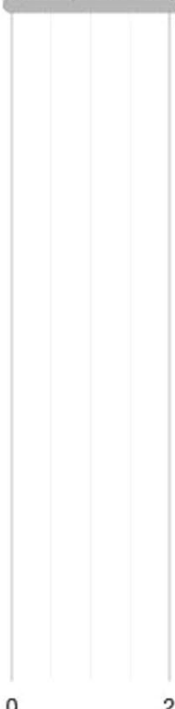

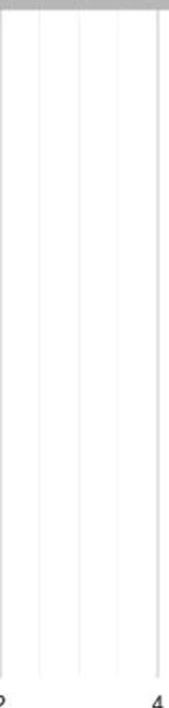

4

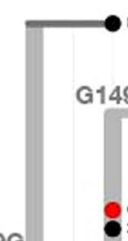

England/CAMM-705B42020/EPI_ISL_441695|20-04-05

- CA-UCSC-912020/20-07-25|Hypothetcal_Farm_9

14948A CA-UCSC-61292920-07-25/Hyposnetcal_Fam _6

A CA-UCSC-5/2020/20-08-05:Hypothetical_Fam_5

CA-UCSC-8/2020|20-07-25|Hypothetcial Farm_ 8

CA-UCSC-3/2020|20-07-22:Hypothetcal-Farm-

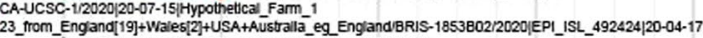

C. 16466 ' EnglandLIVE-A7672/2020|EPI_ISL_517052[20-03-28

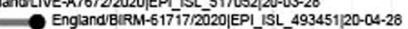

England/201560416042020|EPI_ISL_466311|20-04-07

- England201180398002003

- $13029820-05-13$

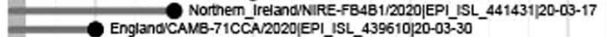

C22232318 Scotland/GCVR-170F94/2020|EPI_ISL_459619|20-03-26

England/BRIS-126510/2020_EPI_ISL_459005120-04-09

-1
1

- England/CAMB-803E2/2020|EPI_ISL_442883|20-04-10

PPUDICNRL_5240/2020|EPIIISL_471550|20-03-23

- Canada/BC_21110314/2020|EPI_ISL_462791|20-03

2 from A England $20144023604 / 2020 \mid$ EPT_ISL_46596/20-03-26

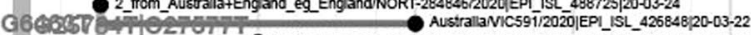

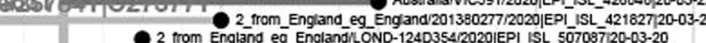

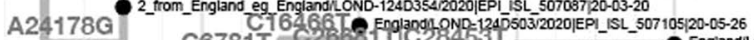

C29364T $6781 \mathrm{~T}$ C2668

EnglanaL OND-124D45120201EPI/ISL-507098|20-04-20

- England/LOND-124D2FD/2020|EPI_ISL_507062|20-04-07

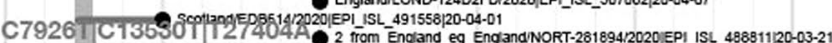

EnglandICAMB-79AC8/2020|EPI_ISL_433820/20-03-26/2F/2020|EPI_ISL_489550|20-03-28

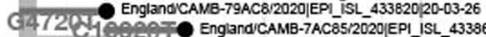

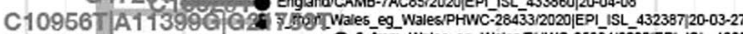

A1440G|A23403G 2 2_from_Wales_eg_Wale6/PHWC-26834/2020/EPI_ISL_422052|20-03-27

T9894G England/CAMB-75BE7/2020|EPI_ISL_438371120-03-28

Wales/PHWC-29138/2020|EPI_ISL_-445485/20-04-04

- Wales/PHWC-23E2B/2020|EPI ISL_418117/20-03-14

14

16

Mutations

Extended Data Fig. 5 | Nextstrain/Auspice view of subtree created by UShER placing the same hypothetical example samples. As in Extended Data Fig.

4. Direct link: https://hextstrain.org/fetch/hgwdev.gi.ucsc.edu/ angie/usher_example.json. 


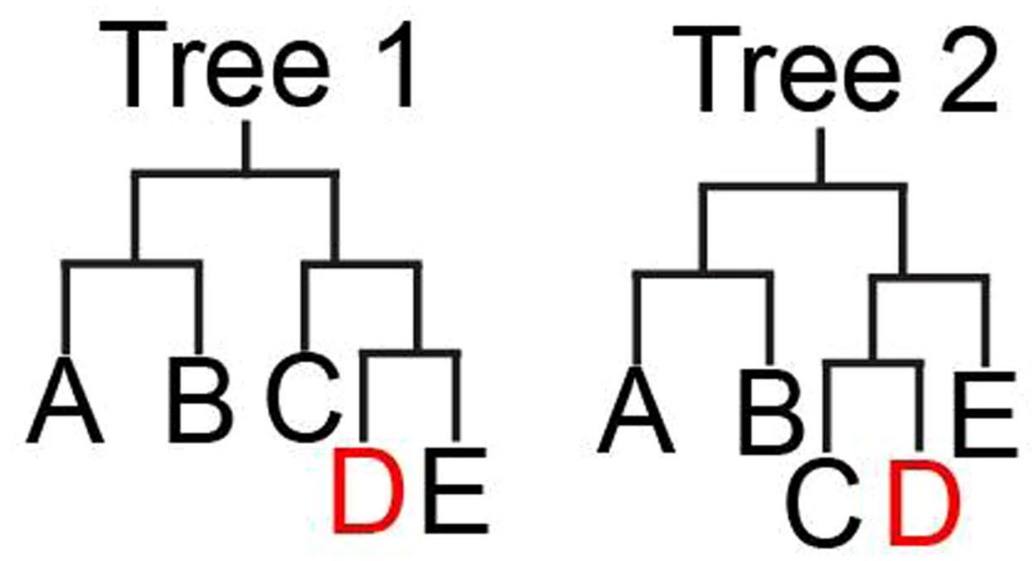

\begin{tabular}{|c|c|c|c|c|}
\hline N1 & Tree 1 Clade & N2 & Tree 2 Clade & N1+N2-2 \\
\hline \multirow{3}{*}{1} & \multirow{3}{*}{$\mathrm{DE}$} & 1 & $\mathrm{CD}$ & $\mathrm{N} / \mathrm{A}$ \\
\cline { 3 - 5 } & & 2 & $\mathrm{CDE}$ & $\mathrm{N} / \mathrm{A}$ \\
\cline { 3 - 5 } & & 3 & $\mathrm{ABCDE}$ & $\mathrm{N} / \mathrm{A}$ \\
\hline \multirow{3}{*}{2} & $\mathrm{CDE}$ & 1 & $\mathrm{CD}$ & N/A \\
\cline { 3 - 5 } & & $\mathbf{2}$ & $\mathrm{CDE}$ & $\mathbf{2}$ \\
\cline { 3 - 5 } & 3 & $\mathrm{ABCDE}$ & $\mathrm{N} / \mathrm{A}$ \\
\hline \multirow{3}{*}{3} & \multirow{3}{*}{$\mathrm{ABCDE}$} & 1 & $\mathrm{CD}$ & N/A \\
\cline { 3 - 5 } & & 2 & $\mathrm{CDE}$ & $\mathrm{N} / \mathrm{A}$ \\
\cline { 3 - 5 } & & $\mathbf{3}$ & $\mathrm{ABCDE}$ & $\mathbf{4}$ \\
\hline
\end{tabular}

Extended Data Fig. 6 | A demonstration of our distance metric for placements. To evaluate the accuracy of each placement in a new phylogeny, we compute the distance for each newly placed sample in the UShER tree (Tree 1) with the reference tree (Tree 2). The clade sets in the two trees are shown for each N1 and N2 value, representing the number of generations from the Sample D in Tree 1 and Tree 2, respectively. We compute the values of $\mathrm{N} 1+\mathrm{N} 2-2$ such that the descendant clades for both trees are identical. In case of newly placed Sample D, clades are identical when N1=2 and N2=2 and when $\mathrm{N} 1=3$ and $\mathrm{N} 2=3$, which are highlighted in bold. Hence the distance (smallest N1+N2-2) from the true placement is equal to 2. 


\section{Reporting Summary}

Nature Research wishes to improve the reproducibility of the work that we publish. This form provides structure for consistency and transparency in reporting. For further information on Nature Research policies, see our Editorial Policies and the Editorial Policy Checklist.

\section{Statistics}

For all statistical analyses, confirm that the following items are present in the figure legend, table legend, main text, or Methods section.

$\mathrm{n} / \mathrm{a}$ Confirmed

$\bigotimes$ The exact sample size $(n)$ for each experimental group/condition, given as a discrete number and unit of measurement

\ A statement on whether measurements were taken from distinct samples or whether the same sample was measured repeatedly

The statistical test(s) used AND whether they are one- or two-sided

Only common tests should be described solely by name; describe more complex techniques in the Methods section.

Х $\square$ A description of all covariates tested

\A description of any assumptions or corrections, such as tests of normality and adjustment for multiple comparisons

$\triangle$ A full description of the statistical parameters including central tendency (e.g. means) or other basic estimates (e.g. regression coefficient) AND variation (e.g. standard deviation) or associated estimates of uncertainty (e.g. confidence intervals)

For null hypothesis testing, the test statistic (e.g. $F, t, r$ ) with confidence intervals, effect sizes, degrees of freedom and $P$ value noted Give $P$ values as exact values whenever suitable.

Х $\square$ For Bayesian analysis, information on the choice of priors and Markov chain Monte Carlo settings

$\bigotimes \square$ For hierarchical and complex designs, identification of the appropriate level for tests and full reporting of outcomes

$\bigotimes \square$ Estimates of effect sizes (e.g. Cohen's $d$, Pearson's $r$ ), indicating how they were calculated

\section{Our web collection on statistics for biologists contains articles on many of the points above.}

\section{Software and code}

Policy information about availability of computer code

Data collection All data used in this work are available from GISAID (gisaid.org), with specific sample accessions listed in Supplemental Files S2-S6. No software was used for data collection.

Data analysis The data was analyzed using code available at https://github.com/yatisht/usher and https://github.com/bpt26/USHER_ANALYSES. We used FastTree version v2.1.10 compiled with double precision, mafft version 7.471, faSplit and faSomeRecords v377, seqmagick commit 0.7.0

+7.g1642bb8, TreeShrink v1.3.7. All software versions are indicated where appropriate in the methods section of the revised manuscript.

For manuscripts utilizing custom algorithms or software that are central to the research but not yet described in published literature, software must be made available to editors and

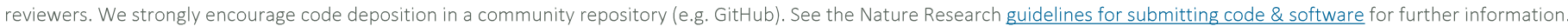

Policy information about availability of data

All manuscripts must include a data availability statement. This statement should provide the following information, where applicable:

- Accession codes, unique identifiers, or web links for publicly available datasets

- A list of figures that have associated raw data

- A description of any restrictions on data availability

All data used in this work are available from GISAID (gisaid.org), with specific sample accessions listed in Supplemental Files S2-S6. 
Please select the one below that is the best fit for your research. If you are not sure, read the appropriate sections before making your selection.

Life sciences

Behavioural \& social sciences

Ecological, evolutionary \& environmental sciences

For a reference copy of the document with all sections, see nature.com/documents/nr-reporting-summary-flat.pdf

\section{Ecological, evolutionary \& environmental sciences study design}

All studies must disclose on these points even when the disclosure is negative.

Study description

In this study, we describe an efficient method that facilitates rapid placement of samples onto an existing phylogeny with applications demonstrated for the current SARS-CoV-2 global phylogeny. We compared our approach to many existing methods and documented accuracy (on simulated data), consistency (with empirical data), compute time and memory usage requirements.

Research sample

Our study is based on existing dataset of SARS-CoV-2 sequences shared via GISAID (gisaid.org). The specific sample accessions are listed in Supplemental Files S2-S6

Sampling strategy

Not relevant. We chose to work primarily with the $11 / 7$ and 31/7 releases of the SARS-CoV-2 phylogeny, because in order to develop our software, we needed a constant tree to perform experiments on and these were the most up-to-date available at the time we began this work. We also worked with simulated data, designed to behave similarly to the real data, as described in our Methods section.

Data collection

All GISAID sequences marked as 'complete' and 'high coverage' submitted up to 31/7/20 were downloaded from GISAID (gisaid.org) and used to build the global phylogeny after a few additional filtering steps (Methods). These data are from a collection of sequences obtained throughout the world during the SARS-CoV-2 pandemic. Tables S2-S6 list all individuals responsible for the primary data collection in all sequences used in this study.

Timing and spatial scale All GISAID sequences starting from EPI_ISL_406798 collected on 26/12/2019 to those deposited in GISAID till 31/7/20 were used. We chose a stopping point of $31 / 7 / 20$ because we needed a consistent sample with which to hone our methods and conduct experiments, as well as to have a "reference tree" to refer back to throughout the study.

Data exclusions

Incomplete and low-coverage sequences as well as those with known issues sequences were excluded (Methods). Our previous study and other related studies cited in the Methods demonstrate that errors can lead to false nucleotide substitutions for myriad reasons unrelated to the biology of the virus itself. We have masked these sites from our analysis and the specific criteria for exclusion are indicated in the method section.

Reproducibility All our findings and results are completely reproducible using the code and data available from https://github.com/yatisht/usher and GISAID (gisaid.org), respectively. Analyses were conducted using code from https://github.com/bpt26/USHER_ANALYSES. Experiments using masking and artificial error were performed with $N=100$ replicates (Figure 2, Extended Data Figure 2). We chose 100 because this is a sufficiently large number of experiments to determine the general behavior of UShER. The experiment for mapping branch parsimony score (Figure 3), viewing multiple trees in DensiTree and comparing two using cophylo (Extended Data Figure 3) and was conducted only once for illustrative purposes, and we randomly chose a sample so that this was representative of the general case. We randomly chose 4 subclades to prune and add back using UShER (Figure 4). To reconstruct subclades (Extended Data Figure 1), we chose $N=5$ as we found five independent subclades of size $\sim 1,000$ that would demonstrate the behavior of each maximum likelihood algorithm in comparison to UShER, and maximum likelihood experiments like this can take a great deal of time.

Randomization Not relevant. We used identical dataset for all comparative analysis hence randomization is not necessary for comparing results of the approaches used in this study.

Blinding

Blinding is not relevant because experimenter bias cannot affect the results of this analysis.

Did the study involve field work? $\square$ Yes $\$ No

\section{Reporting for specific materials, systems and methods}

We require information from authors about some types of materials, experimental systems and methods used in many studies. Here, indicate whether each material, system or method listed is relevant to your study. If you are not sure if a list item applies to your research, read the appropriate section before selecting a response. 
$\mathrm{n} / \mathrm{a}$ Involved in the study $\mathrm{n} / \mathrm{a}$ Involved in the study

\ $\square$ Antibodies

Х $\square$ ChIP-seq

$\square$ Eukaryotic cell lines

Х $\square$ Flow cytometry

Х $\square$ Palaeontology and archaeology

X $\square$ MRI-based neuroimaging

\ Animals and other organisms

\ $\square$ Human research participants

\ $\square$ Clinical data

$\bigotimes \square$ Dual use research of concern 